\title{
Comparing secondary organic aerosol (SOA) volatility distributions derived from isothermal SOA particle evaporation data and FIGAERO-CIMS measurements
}

\author{
Olli-Pekka Tikkanen ${ }^{1,2,3}$, Angela Buchholz ${ }^{1}$, Arttu Ylisirniö ${ }^{1}$, Siegfried Schobesberger ${ }^{1}$, Annele Virtanen ${ }^{1}$, and \\ Taina Yli-Juuti ${ }^{1}$ \\ ${ }^{1}$ Department of Applied Physics, University of Eastern Finland, 70210 Kuopio, Finland \\ ${ }^{2}$ Department of Agricultural Sciences, University of Helsinki, 00790 Helsinki, Finland \\ ${ }^{3}$ Institute for Atmospheric and Earth System Research/Forest Sciences, Faculty of Agriculture and Forestry, \\ University of Helsinki, 00014 Helsinki, Finland
}

Correspondence: Olli-Pekka Tikkanen (olli-pekka.tikkanen@helsinki.fi)

Received: 10 October 2019 - Discussion started: 14 November 2019

Revised: 29 June 2020 - Accepted: 9 July 2020 - Published: 8 September 2020

\begin{abstract}
The volatility distribution of the organic compounds present in secondary organic aerosol (SOA) at different conditions is a key quantity that has to be captured in order to describe SOA dynamics accurately. The development of the Filter Inlet for Gases and AEROsols (FIGAERO) and its coupling to a chemical ionization mass spectrometer (CIMS; collectively FIGAERO-CIMS) has enabled nearsimultaneous sampling of the gas and particle phases of SOA through thermal desorption of the particles. The thermal desorption data have been recently shown to be interpretable as a volatility distribution with the use of the positive matrix factorization (PMF) method. Similarly, volatility distributions can be inferred from isothermal particle evaporation experiments when the particle size change measurements are analyzed with process-modeling techniques. In this study, we compare the volatility distributions that are retrieved from FIGAERO-CIMS and particle size change measurements during isothermal particle evaporation with processmodeling techniques. We compare the volatility distributions at two different relative humidities (RHs) and two oxidation conditions. In high-RH conditions, where particles are in a liquid state, we show that the volatility distributions derived via the two ways are similar within a reasonable assumption of uncertainty in the effective saturation mass concentrations that are derived from FIGAERO-CIMS data. In dry conditions, we demonstrate that the volatility distributions are comparable in one oxidation condition, and in the other oxidation condition, the volatility distribution derived from
\end{abstract}

the PMF analysis shows considerably more high-volatility matter than the volatility distribution inferred from particle size change measurements. We also show that the VogelTammann-Fulcher equation together with a recent glass transition temperature parametrization for organic compounds and PMF-derived volatility distribution estimates are consistent with the observed isothermal evaporation under dry conditions within the reported uncertainties. We conclude that the FIGAERO-CIMS measurements analyzed with the PMF method are a promising method for inferring the volatility distribution of organic compounds, but care has to be taken when the PMF factors are analyzed. Future process-modeling studies about SOA dynamics and properties could benefit from simultaneous FIGAERO-CIMS measurements.

\section{Introduction}

Aerosol particles have varying effects on health, visibility and climate (Stocker et al., 2013). Organic compounds comprise a substantial amount of atmospheric particulate matter (Jimenez et al., 2009; Zhang et al., 2007) of which a major fraction is of secondary origin, i.e., low-volatility organic compounds formed from oxidation reactions between volatile organic compounds (VOCs) and ozone, hydroxyl radicals and nitrate radicals (Hallquist et al., 2009). The aerosol particles containing these kind of oxidation products are called secondary organic aerosols (SOAs) as opposed 
to primary organic aerosols, i.e., organic particles emitted directly to the atmosphere. VOC oxidation reactions result in thousands of different organic compounds (Goldstein and Galbally, 2007). There are gaps in the knowledge, especially on the formation and deposition of SOAs and how the processes are affected by changing physicochemical properties such as volatility (Glasius and Goldstein, 2016). In addition, the phase state of the organic compounds has also been shown to play a role in SOA dynamics (Reid et al., 2018; Shiraiwa et al., 2017; Yli-Juuti et al., 2017; Renbaum-Wolff et al., 2013; Virtanen et al., 2010)

The physicochemical properties of organic aerosols can be studied directly and indirectly. The Aerodyne aerosol mass spectrometer (AMS; Canagaratna et al., 2007; DeCarlo et al., 2006; Jayne et al., 2000) enabled direct and online composition measurements of atmospheric particles for the first time. Combining AMS data with statistical dimension reduction techniques such as factor analysis and positive matrix factorization (PMF; Zhang et al., 2011, 2007, 2005; Paatero and Tapper, 1994) allowed researchers to draw conclusions on sources and types of atmospheric organic particulate matter from the relatively complex mass spectra data.

The chemical ionization mass spectrometer (CIMS; Lee et al., 2014) coupled with the Filter Inlet for Gases and AEROsols (FIGAERO, collectively FIGAERO-CIMS; Lopez-Hilfiker et al., 2014) is a prominent online measurement device for studying both the gas and particle phases of SOAs. During particle-phase measurements, a key advantage over the AMS is the softer chemical ionization that retains much more of the molecular information of the compound than the electron impact ionization used in the AMS. Typically, the collection of the particulate mass is conducted at room temperature, which minimizes the loss of semivolatile compounds during collection. In addition to the overall chemical composition, the gradual desorption of the particulate mass from the FIGAERO filter yields the thermal desorption behavior of each detected ion, i.e., it is a direct measurement of each ion's volatility. FIGAERO-CIMS measurements have been carried out in both laboratory and field environments to study SOA composition from different VOC precursors and in both rural and polluted environments (Le Breton et al., 2018; Huang et al., 2018; Lee et al., 2018; D'Ambro et al., 2017; Lopez-Hilfiker et al., 2015). However, the volatility information in these data sets has barely been used.

Besides direct mass spectrometer measurements, SOA properties have been inferred indirectly from growth (e.g., Pathak et al., 2007 and references therein) and isothermal evaporation (Buchholz et al., 2019; D'Ambro et al., 2018; Yli-Juuti et al., 2017; Wilson et al., 2015; Vaden et al., 2011) measurements. The complexity of organic compounds in these studies can be alleviated with the use of a volatility basis set (Donahue et al., 2006), where organic compounds are grouped based on their (effective) saturation concentration. However, the experimental setup also defines the range of $\mathrm{C}^{*}$ values that can be estimated from the data. Vaden et al. (2011) and Yli-Juuti et al. (2017) have both shown that the volatility basis sets derived from SOA growth experiments result in too fast an SOA evaporation compared to measured evaporation rates when the volatility basis set is used as input for process models. Possible reasons for such discrepancies include the different $\mathrm{C}^{*}$ ranges to which the SOA growth and SOA evaporation experiments are sensitive and the role of vapor wall losses in SOA growth experiments. This raises a need for alternative methods to derive organic aerosol volatility against which the volatilities inferred from the direct particle size measurements can be compared.

Recently, Buchholz et al. (2020) demonstrated that the FIGAERO-CIMS measurements during particle evaporation can be mapped to a volatility distribution of organic compounds by conducting a PMF analysis. On the other hand, Tikkanen et al. (2019) showed that the volatility distribution can be inferred from isothermal particle evaporation measurements by optimizing the evaporation model input to yield the measured evaporation rate at different humidity conditions. In this study, we compare these two approaches for varying oxidation and particle water content conditions. Our main research questions are as follows. (1) Are the volatility distributions derived from particle size change during isothermal evaporation and from the FIGAERO-CIMS measurements similar? (2) How should the PMF results of FIGAERO-CIMS data be interpreted in terms of volatility? (3) Can a recently published glass transition temperature parametrization (DeRieux et al., 2018), combined with the PMF analysis, be used to model particle-phase mass transfer limitations observed for the evaporation in dry conditions, i.e., in the absence of particle-phase water?

\section{Methods}

\subsection{Experimental particle evaporation data}

The experimental data we use are the same as reported in Buchholz et al. $(2019,2020)$. We briefly summarize the measurement setup below. We generated the particles with a potential aerosol mass (PAM) reactor (Kang et al., 2007; Lambe et al., 2011) from the reaction of $\alpha$-pinene with $\mathrm{O}_{3}$ and $\mathrm{OH}$ at three different oxidation levels (average oxygen-to-carbon $(\mathrm{O}: \mathrm{C})$ ratios of $0.53,0.69$ and 0.96$)$. We focus on the lowest $\mathrm{O}: \mathrm{C}(0.53)$ and medium-O : $\mathrm{C}(0.69)$ experiments in this work. The closer analysis of the high- $\mathrm{O}: \mathrm{C}$ experiments suggests particle-phase reactions during the evaporation (Buchholz et al., 2019, 2020). To avoid the uncertainty that would arise from unknown particle-phase reactions, we chose not to include the high-O : $\mathrm{C}$ data in our analysis.

We selected a monodisperse particle population (mobility diameter $d_{\mathrm{p}}=80 \mathrm{~nm}$ ) with two nano-tandem-type differential mobility analyzers (nano-DMA; TSI Incorporated, model 3085) from the initial polydisperse particle population. The 
size selection diluted the gas phase, initiating particle evaporation. The monodisperse aerosol was left to evaporate in a $100 \mathrm{~L}$ stainless-steel residence time chamber (RTC). We measured the particle size distribution during the evaporation with a scanning mobility particle sizer (SMPS; TSI Incorporated, models 3082 and 3775). The RTC filling took approximately $20 \mathrm{~min}$, and we performed the first size-distribution measurement in the middle of the filling interval. To obtain short residence time data (data before $10 \mathrm{~min}$ of evaporation) we added a bypass to the RTC, which led the sample directly to the SMPS. By changing the length of the bypass tubing, we were able to measure the particle size distribution between 2 and $160 \mathrm{~s}$ of evaporation. We measured the isothermal evaporation up to 4-10 h, depending on the measurement. We performed the measurements for each oxidation level both at high relative humidity $(\mathrm{RH}=80 \%)$ and at dry conditions $(\mathrm{RH}<2 \%)$. The change in particle size with respect to time is called an evapogram. In an evapogram, the horizontal axis presents evaporation time, and the vertical axis shows the evaporation factor (EF), i.e., the measured particle diameter divided by the initially selected particle diameter.

To classify the oxidation level of the particles, we derived the average $\mathrm{O}: \mathrm{C}$ ratio from composition measurements with a high-resolution time-of-flight aerosol mass spectrometer (AMS; Aerodyne Research, Inc.). Furthermore, we conducted detailed particle composition measurements with an Aerodyne Research, Inc. FIGAERO (Lopez-Hilfiker et al., 2014) coupled with a chemical ionization mass spectrometer (CIMS), with iodide as the reagent ion (Aerodyne Research Inc.; Lee et al., 2014). Previous studies using FIGAEROCIMS with iodide as the reagent ion found $50 \%$ or better mass closure compared to more established methods of quantifying organic aerosol (OA) mass (albeit with high uncertainties; Isaacman-VanWertz et al., 2017; Lopez-Hilfiker et al., 2016). Therefore, it appears that the bulk of reaction products expected from $\alpha$-pinene oxidation contains the functional groups required for detection by our FIGAEROCIMS.

In the FIGAERO inlet, particles are first collected on a polytetrafluoroethylene (PTFE) filter. Then the collected particulate mass desorbs slowly due to a gradually heated nitrogen flow. The desorbed gaseous compounds are then transported into the CIMS for detection. We derived the average chemical composition of the particles by integrating the detected signal of each ion over the whole desorption interval. For each ion, the change in detected signal with desorption temperature is called a thermogram, and generally, the temperature at the maximum of the thermogram $\left(T_{\max }\right)$ is correlated to the volatility of the detected ion. Similar to Bannan et al. (2019) and Stark et al. (2017), we calibrated the $T_{\max }-$ volatility relationship using compounds with known vapor pressure. The calibration procedure is described in the Supplement.
We collected particles for FIGAERO-CIMS analysis at two different stages of the evaporation. We refer to these samples as either "fresh" or "RTC" samples. The fresh samples were collected for 30 min directly after the selection of the monodisperse population. The RTC samples of the residual particles were collected for $75 \mathrm{~min}$ after $3-4 \mathrm{~h}$ of evaporation in the RTC. The collected particulate mass was 140-260 and 20-70 ng for the fresh and the RTC samples, respectively. More details about sample collection, desorption parameters and data analysis can be found in Buchholz et al. (2020).

\subsection{The volatility distribution}

We represent the myriad of organic compound in the SOA particles with a one-dimensional volatility basis set (1D VBS; below only VBS; Donahue et al., 2006). The VBS groups the organic compounds into "bins" based on their effective (mass) saturation concentration $\mathrm{C}^{*}$, defined as the product of the compounds' activity coefficient and saturation concentration. Generally, a bin in the VBS represents the amount of organic material in the particle and gas phases. In our study, the walls of the RTC have been shown to work as an efficient sink for gaseous organic compounds (Yli-Juuti et al., 2017). Thus, we can assume that the gas phase in our experimental setup does not contain organic compounds, i.e., the amount of organic matter in a bin is the amount in the particle phase. To distinguish from a traditional VBS that groups the organic compounds to bins such that there is a decadal difference in $\mathrm{C}^{*}$ between two adjacent bins, we call the VBS in our work a volatility distribution (VD). We present the amount of material in each VD bin as the dry mole fraction, i.e., the mole fraction of the organics, excluding water. In the analysis presented below, we assign properties to each VD bin (e.g., molar mass), treating each bin as if it consisted of only a single organic compound with a single set of properties. The physicochemical properties of each VD bin are assumed to be the same. These properties and the ambient conditions of each evaporation experiment are listed in $\mathrm{Ta}-$ ble 1 .

\subsection{Deriving volatility distribution from an evapogram}

We followed a similar approach as in Yli-Juuti et al. (2017) and Tikkanen et al. (2019) to derive a VD at the start of the evaporation from an evapogram. To model the evaporation at high $\mathrm{RH}$, we used a process model (liquid-like evaporation model, hereafter LLEVAP) that assumes a liquid-like particle, i.e., a particle in which there are no mass transfer limitations inside the particle and where the mass flux of a VD bin in the particle phase can be calculated directly from the gas-phase concentrations of the VD bin both near the particle surface and far away from the particle (Vesala et al., 1997; Lehtinen and Kulmala, 2003; Yli-Juuti et al., 2017). In this case, the main properties for defining the evaporation rate are 
Table 1. The ambient conditions and properties of the organic compounds used in estimating the VDevap. The variables are, from top to bottom, temperature $(T)$ during the evaporation, relative humidity $(\mathrm{RH})$, gas-phase diffusion coefficient $\left(D_{\mathrm{g}}\right.$, org $)$, molar mass $(M)$, particlephase density $(\rho)$, particle surface tension $(\sigma)$ and mass accommodation coefficient $(\alpha)$. Rows that only have one value are the same in every column.

\begin{tabular}{lrrrr}
\hline Variable & Medium O : C(high RH) & Low O : C(high RH) & Medium O : C(dry) & Low O : C(dry) \\
\hline$T(\mathrm{~K})$ & 293.85 & 293.75 & 293.75 & 293.35 \\
$\mathrm{RH}(\%)$ & 82.4 & 83.5 & 0 & 0 \\
$D_{\text {gas }}^{\mathrm{a}, \mathrm{b}}\left(\mathrm{cm}^{2} \mathrm{~s}^{-1}\right)$ & & & & \\
$M^{\mathrm{b}}\left(\mathrm{g} \mathrm{mol}^{-1}\right)$ & 0.05 & & \\
$\rho^{\mathrm{b}}\left(\mathrm{kg} \mathrm{m}^{-3}\right)$ & 200 & & \\
$\sigma^{\mathrm{b}}\left(\mathrm{mN} \mathrm{m}^{-1}\right)$ & 1200 & \\
$\alpha^{\mathrm{b}}$ & 40 & & \\
\hline
\end{tabular}

${ }^{a}$ The gas-phase diffusion coefficients are scaled to correct the temperatures by multiplying with a factor of $(T / 273.15)^{1.75}$ (Reid et al., 1987).

b Values are chosen to represent a generic organic compound with values similar to other $\alpha$-pinene SOA studies (e.g., Pathak et al., 2007; Vaden et al., 2011; Yli-Juuti et al., 2017).

the saturation concentrations of each VD bin and their relative amounts in the particle.

We used the LLEVAP model to characterize the volatility range that can be interpreted from the evaporation measurements. We calculated the range by modeling the evaporation of a hypothetical particle that consists of one organic compound evaporating in dry conditions. We calculated the evaporation for the range of $\log _{10}\left(\mathrm{C}^{*}\right)$ values from -5 to 5 . We determined the minimum $\mathrm{C}^{*}$ value to be the value that still showed "detectable evaporation", i.e., at least $1 \%$ change in particle diameter during the evaporation time (up to $6 \mathrm{~h}$ ), and the maximum $\mathrm{C}^{*}$ value to be the value before "complete evaporation" occurred, i.e., $99 \%$ particle diameter change within the first $10 \mathrm{~s}$. The minimum $\log _{10}\left(\mathrm{C}^{*}\right)$ calculated with this method was -3 and the maximum $\log _{10}\left(\mathrm{C}^{*}\right)$ was 2 . We then modeled the particle composition with six VD bins with $\mathrm{C}^{*}$ values between these minimum and maximum values. Each VD bin has a decadal difference in $\mathrm{C}^{*}$ to an adjacent VD bin (like in the traditional VBS). We note that, based on this analysis, all the compounds with $\log _{10}\left(C^{*}\right)<-3$ will not evaporate during the experimental timescale. This means that any compounds with lower $\mathrm{C}^{*}$ than this threshold will be assigned to the $\log _{10}\left(C^{*}\right)=-3$ VD bin. Similarly, any compound with $\log _{10}\left(\mathrm{C}^{*}\right)>2$ will be classified into the $\log _{10}$ $\left(C^{*}\right)=2$ VD bin or not be detected at all due to evaporating almost entirely before the first measurement point.

We calculated the dry particle mole fraction of each VD bin at the start of the evaporation by fitting the evaporation predicted with the process model to the measured evapograms. Our goal was to minimize the mean squared error in a vertical direction between the experimental data and the LLEVAP output. We used the Monte Carlo genetic algorithm (MCGA; Berkemeier et al., 2017; Tikkanen et al., 2019) for the input optimization. In the optimization, we set the population size to be 400 candidates, number of elite members to
20 (5\% of the population), number of generations to 10 and number of candidates drawn in the Monte Carlo (MC) part to 3420, which corresponds to half of the total process model evaluations done during the optimization. We performed the optimization 50 times for each evapogram and selected the best-fit VD estimate for further analysis.

The VD derived from the evapogram is hereafter referred to as the $\mathrm{VD}_{\text {evap. }}$. The initial composition of the SOA particles in the dry and wet experiments was the same and can be described by the same fitted $\mathrm{VD}_{\text {evap }}$ as the particles were generated at the same conditions in the PAM and only the evaporation conditions changed.

\subsection{Deriving volatility distribution from FIGAERO-CIMS measurement}

As shown by Bannan et al. (2019) and Stark et al. (2017), the peak desorption temperature, $T_{\max }$, can be used together with a careful calibration to link desorption temperatures from the FIGAERO filter to $\mathrm{C}^{*}$ values for the detected ions. In principle, this would allow us to assign one $\mathrm{C}^{*}$ value to each ion thermogram. But this assumes that one detected ion characterized by its exact mass is indeed just one compound. In practice, this is not always the case, and for some ion thermograms, a bimodal structure or distinct shoulders and/or broadening is visible. This can be caused by isomers of different volatility which cannot be separated even by highresolution mass spectra.

Another complication arises due to the thermal-desorption process delivering the collected aerosol mass into the CIMS. Especially multifunctional and, hence, low-volatility compounds may thermally decompose before they desorb from the filter and, thus, are detected as smaller ions. The apparent desorption temperature is then determined by the thermal stability of the compound and not its volatility. Typically, this decomposition process starts at a minimum temperature and 
will not create a well-defined peak shape (Buchholz et al., 2020, Schobesberger et al., 2018), presumably because an observed decomposition product may have multiple sources, especially when including all isomers, and the ion signal for the respective composition may overlap with the signal of isomers derived from true desorption. For example, a true constituent of the SOA particle may give rise to an observed main thermogram peak, but it may be broadening and/or tailing if a decomposition product has the same composition. By ignoring this and simply using the $T_{\max }$ values, the true volatility of the SOA particle constituents will be overestimated, i.e., the derived VD will be biased towards higher $\mathrm{C}^{*}$ bins.

One more potential source of bias is our implicit assumption of a constant sensitivity of the CIMS towards all compounds, which follows from the lack of calibration measurements for our data sets (which indeed is a challenging endeavor; e.g., Isaacman-VanWertz et al., 2018). It is plausible that less volatile compounds tend to be detected at higher sensitivity (Iyer et al., 2016; Lee et al., 2014), up to a kinetic limit sensitivity. Consequently, a volatility distribution derived from FIGAERO-CIMS thermograms may be biased towards lower volatility ( $\mathrm{C}^{*}$ bins), at least for compositions not associated with thermal decomposition.

To separate the multiple sources possibly contributing to each ion thermogram (isomers and thermal decomposition products), we applied the positive matrix factorization (PMF; Paatero and Tapper, 1994) to the FIGAERO-CIMS data set. PMF is a well-established mathematical technique in atmospheric science mostly used to identify the contribution of different sources of aerosol particle constituents or trace gases in the atmosphere. PMF represents the measured matrix of the time series of mass spectra, $\mathbf{X}$, as a linear combination of a known (or unknown) number of constant source profiles, $\mathbf{F}$, with varying contributions over time, $\mathbf{G}$, as follows:

$\mathbf{X}=\mathbf{G} \cdot \mathbf{F}+\mathbf{E}$.

$\mathbf{E}$ is a matrix containing the residuals between the measured $(\mathbf{X})$ and the fitted data $(\mathbf{G} \cdot \mathbf{F})$. Values for $\mathbf{G}$ and $\mathbf{F}$ are found by minimizing this residual, $E_{\boldsymbol{i} j}$, scaled by the corresponding measurement error, $S_{i j}$, for each ion $i$ at each time $j$ as follows:

$Q=\sum_{i=1}^{m} \sum_{j=1}^{n}\left(\frac{E_{i, j}}{S_{i, j}}\right)$.

Each row in $\mathbf{F}$ contains a factor mass spectrum and each column in $\mathbf{G}$ holds the corresponding time series of contribution by each factor. In the case of FIGAERO-CIMS data, the time series is equivalent to the desorption temperature ramp during the thermogram and will be called the "mass loading profile" below. The absolute values (temperature or time) are irrelevant for the performance of PMF as the " $x$ values" are only used to determine the order of the data points but have no influence on the model output (Paatero and Tapper, 1994). This allowed us to combine multiple separate thermogram measurements into one data set and conduct a PMF analysis. This simplified the comparison of factors between measurements. More details about the PMF method in the specific case of FIGAERO-CIMS data can be found in Buchholz et al. (2020).

Once the PMF algorithm was applied to the FIGAEROCIMS data, we calculated the VD from the mass loading matrix G. Due to the very low signal strength of many ions, the CIMS data had been averaged over $20 \mathrm{~s}$, leading to an enhanced reliability of the high-resolution analysis. This leads to an average desorption temperature difference $\Delta T_{\text {desorp }} \approx$ $4{ }^{\circ} \mathrm{C}$ between two adjacent data points. To overcome this coarse $T_{\text {desorp }}$ grid, we interpolated each factor's mass loading profile with a resolution of 100 sample points between two temperature steps to gain sufficient statistics for further analysis. $T_{\max }$ was determined as the temperature at the maximum signal in the factor mass loading profile. We integrated the factor mass loading profile and defined the temperatures where the value of the integral reached $25 \%$ and $75 \%$ of its maximum value. This temperature interval formed the factor's desorption temperature range, and the corresponding $\mathrm{C}^{*}$ values will be used in Sect. 3.3. We converted the $T_{\max }$ values into $C^{*}$ values and the desorption temperature range into a $\mathrm{C}^{*}$ range with a parametrization derived from calibration measurements (see the Supplement for details) with organic compounds with known $\mathrm{C}^{*}$ values.

$\mathrm{C}^{*}=\frac{\exp \left(\alpha+\beta T_{\text {factor }}\right) M_{\text {org }}}{R T_{\text {ambient }}} 10^{9}$,

where $\mathrm{C}^{*}$ is the effective saturation concentration in units $\mu \mathrm{g} \mathrm{m}^{-3}, M_{\text {org }}$ is the molar mass of the organic compound assumed to be $M_{\text {org }}=0.2 \mathrm{~kg} \mathrm{~mol}^{-1}, R$ is the universal gas constant, $T_{\text {factor }}$ (in ${ }^{\circ} \mathrm{C}$ in Eq. 3 ) is the temperature of the mass loading profile and $T_{\text {ambient }}$ (in Kelvin in Eq. 3 ) is the ambient temperature at which the evaporation happens (see Table 1). $\alpha$ and $\beta$ are the fitted coefficients from the calibration data, where $\alpha=(-1.431 \pm 0.31)$ and $\beta=(-0.207 \pm 0.006){ }^{\circ} \mathrm{C}^{-1}$. We applied the lower and higher bounds of the fitting coefficients' uncertainty when we calculated the $\mathrm{C}^{*}$ range in Sect. 3.3. Finally, the signal fraction of each factor was calculated by dividing the integral of a factor's signal over the whole temperature range with the sum of integrals of all factors. We compare this signal fraction to the dry mole fraction in the $\mathrm{VD}_{\text {evap. }}$. We refrained from converting the counts per second signal into moles as no adequate transmission and sensitivity measurements were available for the FIGAEROCIMS setup used. We refer to the volatility distribution, calculated from the PMF data using the $T_{\max }$ values of each factor as VDPMF, later in this work.

With Eq. (3) we can calculate the minimum and maximum $\mathrm{C}^{*}$ values that can be resolved from a FIGAERO thermogram. The desorption temperature was ramped between 27 and $200^{\circ} \mathrm{C}$, but defined peaks (and thus $T_{\max }$ values) can be 
detected only between 30 and $180^{\circ} \mathrm{C}$. Thus, the resolvable $\log _{10}\left(C^{*}\right)$ values range from 1.6 to -11.9 . It has to be kept in mind that, strictly speaking, this calibration only applies to the $T_{\max }$ values of a single ion thermogram.

\subsection{Modeling particle viscosity at dry conditions}

To model the mass transfer limitations observed in the evaporation measurements at dry conditions (Buchholz et al., 2019), we used the kinetic multilayer model for gas particle interactions (KM-GAP; Shiraiwa et al., 2012), with modifications described in Yli-Juuti et al. (2017) and Tikkanen et al. (2019). The main modification to the original model was that, during evaporation, the topmost layer (the quasistatic surface layer) merged with the first bulk layer if the thickness of the layer was smaller than $0.3 \mathrm{~nm}$. We calculated the viscosity at each layer of the particle as follows:

$\log _{10}\left(\eta_{j}\right)=\sum_{i=1}^{N} X_{\text {mole }, i, j} \log _{10}\left(b_{i}\right)$,

where $X_{\text {mole }, i, j}$ is the mole fraction of the VD bin $i$ in layer $j$, and $b_{i}$ is a coefficient that describes the contribution of each VD bin to the overall viscosity.

Since we generated the particles in the same environment (PAM chamber) and only the evaporation happened at different conditions, the VD at the start of the evaporation derived from high-RH data also represents the composition at the start of the evaporation in dry conditions. Then we can use the best fit $\mathrm{VD}_{\text {evap }}$ from the high-RH data as input for KM-GAP and fit the $b_{i}$ values in Eq. (4) to the dry data set. We set the minimum and maximum allowed values for $b_{i}$ to $10^{-15}$ and $10^{20}$, respectively. To estimate the $b_{i}$ values when modeling the evaporation with $\mathrm{VD}_{\mathrm{PMF}}$ in dry conditions, we calculated these $b_{i}$ terms using the mass spectra of each factor (F in Eq. 1) and the Vogel-Tammann-Fulcher (VTF) equation (DeRieux et al., 2018; Angell, 2002, 1995) as follows:

$\eta_{i}=\eta_{\infty} \exp \left(\frac{T_{0, i} D}{T-T_{0, i}}\right)$,

where $\eta_{i}$ is the viscosity of the $i$ th VD bin and/or PMF factor. $\eta_{i}$ can be seen as a proxy for $b_{i}$ in an ideal solution. $\eta_{\infty}$ is the viscosity at infinite temperature, $T_{0, i}$ is the Vogel temperature of the $i$ th VD bin and $D$ is a fragility parameter. Setting $\eta_{\infty}=10^{-5}$ and $\eta\left(T_{\mathrm{g}}\right)=10^{12} \mathrm{~Pa} \mathrm{~s}^{-1}$ (e.g., DeRieux et al., 2018; Gedeon, 2018), where $T_{\mathrm{g}}$ is the glass transition temperature of a compound, yields the following:

$T_{0, i} \approx \frac{39 \cdot 14 T_{\mathrm{g}, i}}{39.14+D}$.

We calculated $T_{\mathrm{g}}$ for every compound in the PMF mass spectra with a parametrization for SOA matter developed by DeRieux et al. (2018). This parametrization requires the number of carbon, oxygen and hydrogen atoms to calculate the $T_{\mathrm{g}}$. We then computed $T_{\mathrm{g}}$ for each PMF factor as a mass fraction weighted sum of the glass transition temperatures of individual compounds (DeRieux et al., 2018; Dette et al., 2014). Based on the $T_{\mathrm{g}, i}$ of each PMF factor, we calculated the viscosity of each PMF factor with Eqs. (5) and (6) and used them as an approximation for $b_{i}$. We used fragility parameter value $D=10$ in the calculations, according to DeRieux et al. (2018).

\section{Results}

In this section we first focus on the high-RH experiments in which evaporation is modeled with the LLEVAP model. We will first compare VDevap to VDPMF for which the $C^{*}$ of a PMF factor is determined from the factor's $T_{\max }$ value. Then, we compare the volatility distributions where the $\mathrm{C}^{*}$ of a PMF factor is determined as a range from the 25th and 75th percentile desorption temperatures. Lastly, we study the volatility distributions in dry conditions. We investigate the VD on both a qualitative and quantitative level. On a qualitative level, we compare the amount of matter of different $\mathrm{C}^{*}$ intervals relevant for the evaporation process. On a quantitative level, we study what the evaporation behavior of the particles is based on the determined VD and how they compare to the measured evaporation.

\subsection{PMF solution interpretation}

Figure S2 in the Supplement shows the mass loading profiles derived from the FIGAERO-CIMS measurements of medium- and low-O : C particles at high $\mathrm{RH}$. The corresponding factor mass spectra can be found in Figs. S3 and S4 in the Supplement. A key step in any PMF analysis is determining the "right" number of factors as this can affect the interpretation of the results. We carefully investigated the $Q / Q_{\exp }$, the time series of scaled and unscaled residuals, and the ability of a PMF solution to capture the characteristic behavior of as many single ion thermograms as possible (see Buchholz, 2020, for details). Based on this analysis, a seven-factor solution was chosen for the medium-O : $\mathrm{C}$ cases and a nine-factor solution for the low-O : $\mathrm{C}$ ones. The two additional factors in the low- $\mathrm{O}: \mathrm{C}$ case were needed to capture a contamination on the FIGAERO filter during the dry, fresh sample (factors LC1 and LC2 in Figs. S2 and S4). As these two factors were clearly an artifact introduced by the FIGAERO filter sampling, we omitted their contribution for the following analysis. From careful comparison of the factor profiles and mass spectra with filter blank measurements, we determined that factor $\mathrm{MB} 1$ in the medium- $\mathrm{O}: \mathrm{C}$ case and factor LB1 in the low-O : C case describe the filter and/or instrument background and are thus also excluded from the VD comparison presented below.

Factors $1-5$ in both $\mathrm{O}: \mathrm{C}$ cases exhibit a monomodal peak shape and can thus be characterized by their $T_{\max }$ values. Factor MD1 in the medium-O : $\mathrm{C}$ case and factor 

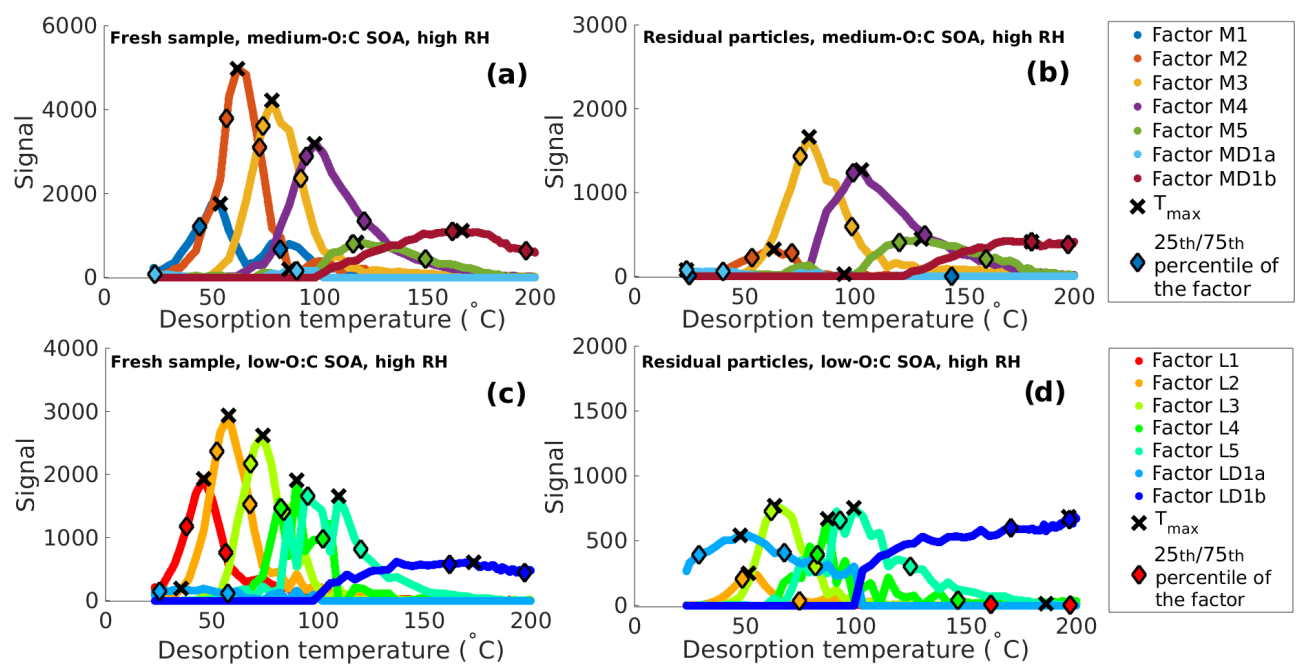

Figure 1. Main positive matrix factorization (PMF) mass loading profiles for the thermal desorption of secondary organic aerosol (SOA) from $\alpha$-pinene at high relative humidity (RH) conditions. (a) Fresh sample of medium-O : C SOA. (b) Residual particles of medium-O : C SOA after 173-259 min of evaporation in a residence time chamber (RTC; the RTC sample). (c) Fresh sample of low-O : C SOA. (d) Residual particles of low-O : C SOA after 168-254 min of evaporation in the RTC (the RTC sample). Black crosses indicate the peak desorption temperature $T_{\max }$, and the diamonds mark the 25 th and 75 th percentiles of the area of each factor.

LD1 in the low-O : $\mathrm{C}$ case need to be investigated more closely, as their factor mass spectrum and the sometimes bimodal mass loading profile suggest that these factors contain compounds stemming from both direct desorption (desorption $T<100^{\circ} \mathrm{C}$ ) and thermal decomposition (desorption $T>100^{\circ} \mathrm{C}$; see Buchholz et al., 2020, for details). To account for this, the factor is split into two, with the first half containing the signal from desorption temperatures below $100{ }^{\circ} \mathrm{C}$ (factor $\mathrm{M} / \mathrm{LD} 1 \mathrm{a}$ ) and the second half containing temperatures above $100^{\circ} \mathrm{C}$ (factor $\mathrm{M} / \mathrm{LD} 1 \mathrm{~b}$ ). We treat these factors separately. We note that now the latter half of the split factor is dominated by thermal decomposition products, so the apparent desorption temperature is actually the temperature at which thermal decomposition leads to products which desorb at this temperature. This apparent desorption temperature is thus a lower limit for the decomposing parent compound, i.e., the true volatility of these parent compounds is even lower. However, the desorption temperatures are so high that they lead to $\log _{10}\left(\mathrm{C}^{*}\right)<-3$ and are thus below the comparable range for $\mathrm{VD}_{\text {evap }}$. Figure 1 (high-RH data) and Fig. S10 in the Supplement (dry-condition data) show the mass loading profiles derived from FIGAERO-CIMS measurements of medium- and low-O : C particles after we excluded the contamination and background factors and split the decomposition factors.

\subsection{Volatility distribution comparison at high RH based on factor $T_{\max }$}

To compare $\mathrm{VD}_{\text {evap }}$ and $\mathrm{VD}_{\mathrm{PMF}}$, we need to determine the time interval in the evapogram that the VDPMF represents. We collected the fresh samples directly after the size selec- tion. As the particles were collected for $30 \mathrm{~min}$, the collected sample represents particles that have evaporated from 0 up to $30 \mathrm{~min}$ in the organic vapor-free air. We note that this is different from the standard FIGAERO-CIMS sample collection in which the particles are collected in a quasi-equilibrium with the surrounding gas phase, and no significant evaporation occurs (Lopez-Hilfiker et al., 2014). For RTC samples, we also need to consider that not all particles have evaporated for the same time due to the filling of the RTC for ca. 20 min. We determined the minimum time the particles have evaporated in the RTC as the time when we started the sample collection minus the RTC filling time. We determined the maximum evaporation time in the RTC to be the time when we stopped the sample collection plus the filling time. These minimum and maximum comparison times are shown in Table S1 in the Supplement, and they are referred to as minimum and maximum (sample) evaporation time. The mean (sample) evaporation time is defined as being at the middle of the sample collection interval. For simplicity, we will show, in the main text, the results from the analysis in which the FIGAERO-CIMS samples were assumed to represents the particles at the mean sample evaporation time. We show the analysis in which the samples were assumed to represent the particles at minimum and maximum evaporation time in the Supplement. The choice of sample evaporation time does not affect the conclusions we draw about the analysis presented in this section.

Figure 2 shows $\mathrm{VD}_{\text {evap }}$ and $\mathrm{VD}_{\mathrm{PMF}}$ for medium- (Fig. 2ab) and low-O : C (Fig. 2c-d) particles in high-RH experiments. In the VDPMF calculated from the $T_{\max }$ value of each factor (black crosses), the factors fall into three dif- 

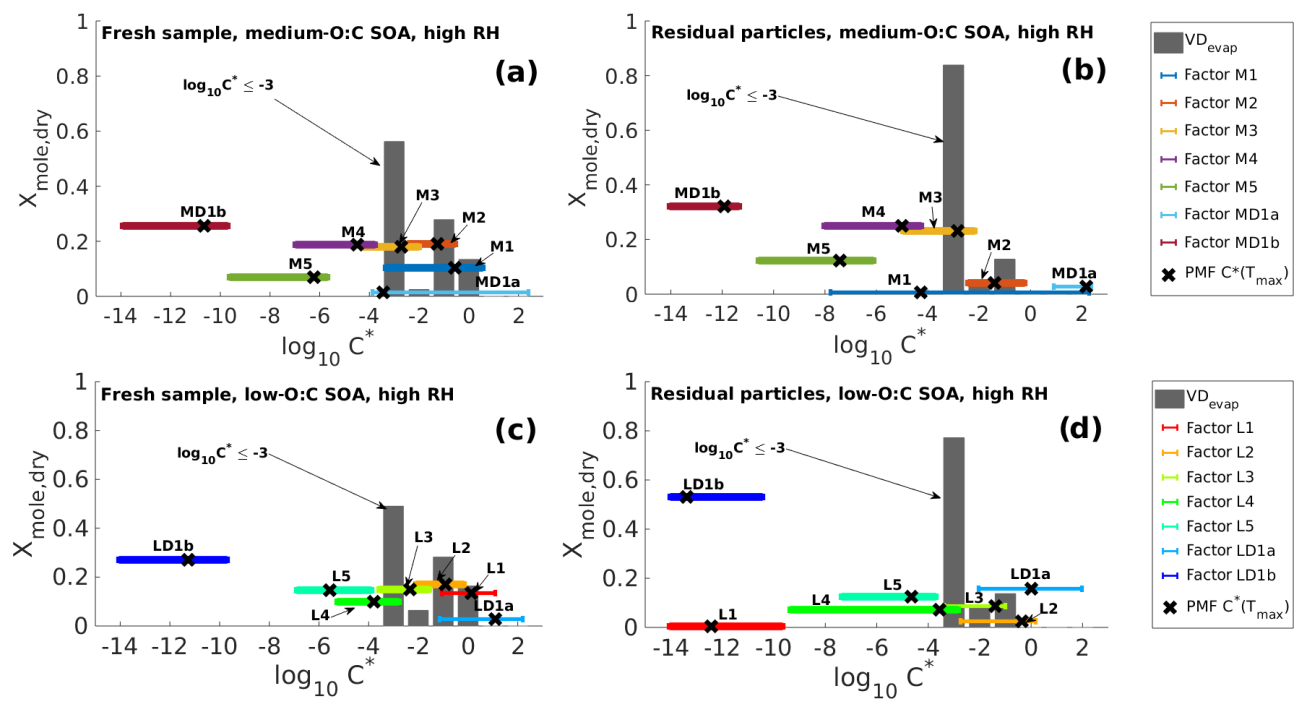

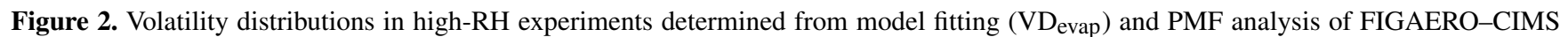
data (VDPMF) for the same four cases as shown in Fig. 1. (a) Fresh sample of medium-O : C SOA. (b) Residual particles of medium-O : C SOA (the RTC sample). (c) Fresh sample of low-O : C SOA. (d) Residual particles of low-O : C SOA (the RTC sample). VDevap is shown for the best-fit simulation (gray bars) at the mean evaporation time of the FIGAERO-CIMS sample. Black crosses show the $\log _{10}\left(\mathrm{C}^{*}\right) \mathrm{calculated}$ for each PMF factor from the peak desorption temperature $T_{\max }$. The horizontal colored lines show the range of $\log _{10}\left(\mathrm{C}^{*}\right)$ calculated from the 25th and 75th percentiles of each PMF factor's mass loading profile.

ferent volatility classes within our chosen particle size and experimental timescale, namely practically nonvolatile $\left(\log _{10}\left(C^{*}\right) \leq-2\right)$, slightly volatile $\left(-2<\log _{10}\left(C^{*}\right) \leq 0\right)$ and volatile $\left(\log _{10}\left(\mathrm{C}^{*}\right)>0\right)$. We use these three volatility classes to compare the volatility distributions in Fig. 3 where each VD bin is grouped to these three volatility classes. Figure 3 compares $\mathrm{VD}_{\mathrm{PMF}}$ to what $\mathrm{VD}_{\text {evap }}$ is at the mean time that the FIGAERO samples had evaporated prior to collection. We show the same comparison for the minimum and maximum evaporation time in Fig. S6 in the Supplement.

After the volatility class grouping is applied, we see that there are differences between $V_{\text {evap }}$ and VDPMF. With VDPMF of the fresh samples, there are excess amounts of matter in the lowest volatility class (volatility class 1 ) and less material in volatility class 2 compared to $\mathrm{VD}_{\text {evap }}$ for both oxidation conditions. In addition, the $\mathrm{VD}_{\mathrm{PMF}}$ of the low-O : $\mathrm{C}$ fresh sample shows more material in the highest volatility class (volatility class 3 ) compared to $\mathrm{VD}_{\text {evap }}$.

To investigate the observed discrepancies further, we used the VD $D_{\mathrm{PMF}}$ shown in Fig. 2 as an input to the LLEVAP model and calculated the corresponding isothermal evaporation behavior (i.e., the evapogram). We show these simulated evapograms in Fig. 4a for the medium-O : C case and in Fig. 4b for the low-O : C condition together with the simulated evapogram calculated using $\mathrm{VD}_{\text {evap }}$ as an input for the LLEVAP model. The simulated evapograms calculated with the VDPMF of the fresh samples do not match the measured evapograms, while the evapogram calculated with $\mathrm{VD}_{\text {evap }}$ agrees well with the experimental evapogram (black lines in Fig. 4), as expected, since this is the goal of the $\mathrm{VD}_{\text {evap }}$ determina- tion. The simulation calculated with the VDPMF of the fresh sample (light blue lines in Fig. 4 for the mean evaporation time; Fig. S7 in the Supplement for other evaporation times) shows slower evaporation than the observations or the simulation calculated with $\mathrm{VD}_{\text {evap }}$. This is consistent with the results show in Fig. 3, where the VD $\mathrm{PMF}_{\mathrm{P}}$ contained more lowvolatility material than the $\mathrm{VD}_{\text {evap }}$.

Figure 4 also shows the simulated evapograms calculated with VDPMF of the RTC samples (light brown lines in Figs. 4 and S7). In these cases, the particles size decreases little within the simulation timescale. With medium-O : C particles, the simulated evaporation matches the measured evaporation well. With low-O : C particles, the evaporation calculated with VDPMF is too fast. The shape of the evapogram does not match the measured one.

\subsection{Applying desorption range to characterize the volatility of PMF factors}

The $T_{\max }$ value is a practical choice for the characteristic temperature of the desorption process. However, as we saw in Sect. 3.2, the VDPMF calculated from the peak desorption temperatures did not produce the measured evapogram when used as an input for the LLEVAP model. Working under the assumption that all material collected on the FIGAERO filter, including the higher volatility material, is detected in the CIMS and then captured in the PMF analysis, we will relax the assumption that the volatility of the factor is characterized strictly by the $T_{\max }$ value of the factor and investigate the VDPMF further. We will explore how the 

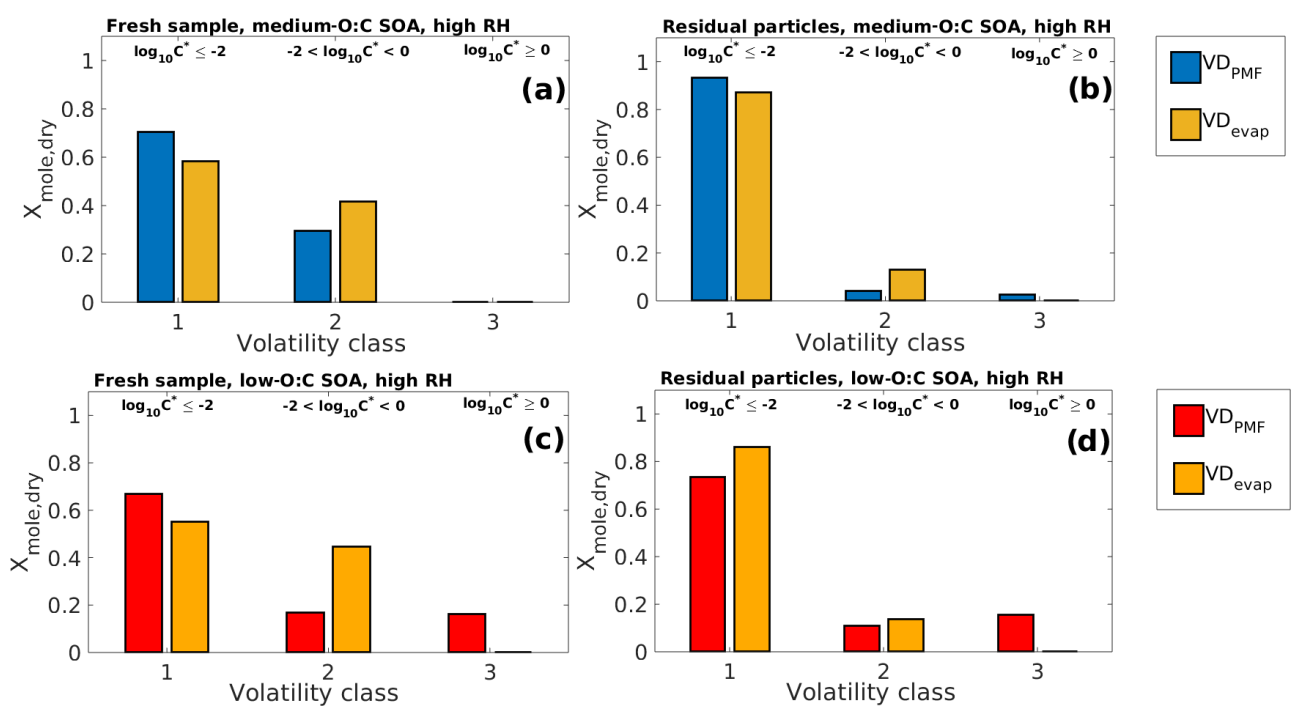

Figure 3. Comparison of $\mathrm{VD}_{\mathrm{PMF}}$ and $\mathrm{VD}_{\mathrm{evap}}$ at the mean sample evaporation time in high-RH experiments for the same four cases as shown in Fig. 1. (a) Fresh sample of medium-O : C SOA. (b) Residual particles of medium-O : C SOA (the RTC sample). (c) Fresh sample of lowO : C SOA. (d) Residual particles of low-O : C SOA (the RTC sample). The VD bins shown in Fig. 2 are grouped into three different volatility classes based on their evaporation tendency with respect to the measurement timescale and particle size. The limits for each volatility class are shown at the top and are the same for each subfigure. The VDPMF shows lower overall volatility than the VDevap, except for (d) (RTC sample of low-O : C SOA) where the VDPMF shows higher overall volatility than the VDevap.
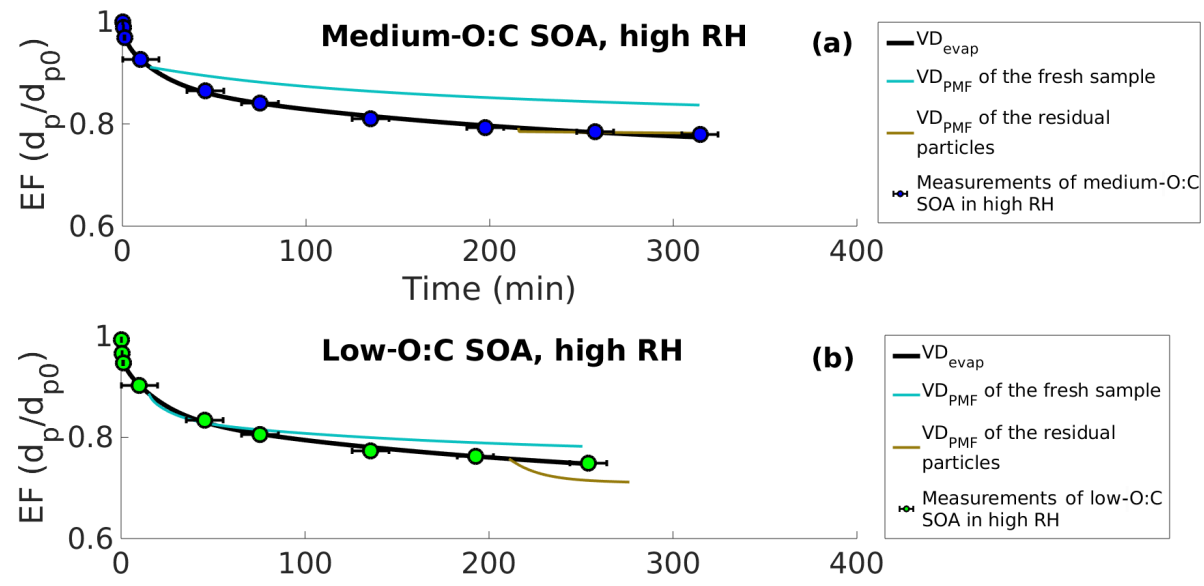

Figure 4. Evapograms of high-RH experiments showing the evaporation factors (remaining fraction of the initial particle diameter; circles) and their uncertainty in time for (a) medium-O : C SOA and (b) low-O : C SOA. Liquid-like evaporation model (LLEVAP) simulated evapograms calculated using the best fit $\mathrm{VD}_{\text {evap }}$ (solid black lines) and LLEVAP simulated evapograms calculated with VDPMF (turquoise lines for VDPMF of fresh SOA and light brown lines for simulations with VDPMF of the residual particles evaporated for 173-259 and 168-254 min for medium- and low-O : C SOAs, respectively). The evapograms calculated with the VDPMF of the fresh samples show a lower rate of evaporation than the evapogram calculated with the $\mathrm{VD}_{\text {evap, }}$, which is consistent with volatility distribution shown in Fig. 3. The evapograms calculated with the VDPMF of the residual particles (the RTC sample) show a similar rate of evaporation for medium-O : C SOA and a faster rate of evaporation for low-O : C SOA compared to evapograms calculated with VDevap, which is similarly consistent with Fig. 3.

VDPMF changes when the desorption temperature and the resulting $\mathrm{C}^{*}$ are interpreted to contain uncertainty and if the VDPMF, considering these uncertainty ranges, is consistent with the observed isothermal evaporation. The uncertainty in the desorption temperature raises from the fact that com- pounds volatilize from the FIGAERO filter throughout the heating, and therefore, one value might not be adequate to characterize the $\mathrm{C}^{*}$ of a factor and each PMF factor contains multiple compounds with distinct $\mathrm{C}^{*}$. 
Table 2. The best-fit $\mathrm{C}^{*}$ values for medium- and low-O : $\mathrm{C}$ high-RH experiments, when $\mathrm{C}^{*}$ values of PMF factors were optimized with respect to the measured isothermal evaporation. $\mathrm{C}^{*}$ values were optimized by assuming that the FIGAERO-CIMS sample represents particle composition at the mean sample evaporation time for the fresh sample and the minimum sample evaporation time for the RTC sample. The $\mathrm{C}^{*}$ values are rounded to two significant digits and are in units $\mu \mathrm{g} \mathrm{m}^{-3}$. $\mathrm{C}^{*}$ values below $10^{-3} \mu \mathrm{g} \mathrm{m}^{-3}$ are not reported explicitly since the evapogram-fitting method is not sensitive to these values.

\begin{tabular}{lrrrr}
\hline & Medium O : C (fresh sample) & Medium O : C (RTC sample) & Low O : C (fresh sample) & Low O : C (RTC sample) \\
\hline Factor M1/L1 & $4.96 \times 10^{-1}$ & 36.10 & $3.06 \times 10^{-1}$ & $<10^{-3}$ \\
Factor M2/L2 & $2.89 \times 10^{-1}$ & $4.12 \times 10^{-1}$ & $3.55 \times 10^{-1}$ & $2.40 \times 10^{-2}$ \\
Factor M3/L3 & $9.93 \times 10^{-3}$ & $4.42 \times 10^{-3}$ & $2.87 \times 10^{-2}$ & $7.13 \times 10^{-3}$ \\
Factor M4/L4 & $<10^{-3}$ & $<10^{-3}$ & $1.54 \times 10^{-3}$ & $<10^{-3}$ \\
Factor M5/L5 & $<10^{-3}$ & $<10^{-3}$ & $<10^{-3}$ & 130.03 \\
Factor D1a & $7.68 \times 10^{-1}$ & 69.35 & $<10^{-3}$ & $1.04 \times 10^{-1}$ \\
Factor D1b & $<10^{-3}$ & $<10^{-3}$ & $<10^{-3}$ \\
\hline
\end{tabular}

We calculated the 25th and 75th percentiles of the desorption temperatures of each factor and converted them to effective saturation concentrations, as described in Sect. 2.4 (see diamond markers in Fig. 1). We show the resulting $\mathrm{C}^{*}$ ranges in Fig. 2 as solid horizontal lines, where the line color matches the color of the factors in Fig. 1. We then ran the MCGA optimization by setting the number of compounds equal to the number of PMF factors, the molar fraction for each compound at the FIGAERO-CIMS sampling time fixed to the molar fraction of the corresponding factor and the $\mathrm{C}^{*}$ as the optimized variables restricted to the range corresponding to the 25th and 75th percentile desorption temperature. In the optimization, the goodness-of-fit statistic was calculated as a mean squared error, similar to the determination of $\mathrm{VD}_{\text {evap. }}$.

As the fresh samples were collected between 0 and $30 \mathrm{~min}$ from the start of the evaporation, we sought a fitting set of $\mathrm{C}^{*}$ values for evaporation starting at 0,15 and $30 \mathrm{~min}$. Again, we show the results for the mean sample evaporation time (15 min) in the main text and the results for the other evaporation times in the Supplement. Due to scarcity of particle size measurements at the collection time of the RTC sample, we will apply this analysis only to the VDPMF of the RTC sample at its minimum evaporation time. In each optimization, we set the initial particle diameter to be the same as what is simulated with $\mathrm{VD}_{\text {evap }}$. We derived $50 \mathrm{C}^{*}$ estimates for both samples. From these 50 estimates, we chose the best-fit evapogram. We refer to these optimized volatility distributions as $\mathrm{VD}_{\mathrm{PMF}}$,opt to separate them from the VDPMF where we used $T_{\max }$ to characterize $\mathrm{C}^{*}$ of a PMF factor.

We show the optimized $C^{*}$ values forming VDPMF,opt in Table 2 (see Table S2 in the Supplement for results with minimum and maximum sample evaporation times). Figure 5 shows the best-fit evaporation simulations calculated with VDPMF,opt. The other sample evaporation times are displayed in the Supplement (Fig. S8). For both oxidation conditions, the simulations resemble the experimental evapogram and the evapogram calculated with $\mathrm{VD}_{\text {evap }}$, al- though the simulation of the medium- $\mathrm{O}: \mathrm{C}$ condition shows a 5 times larger goodness-of-fit value compared to the simulation calculated with $\mathrm{VD}_{\text {evap }}$. The evapograms determined with the VDPMF,opt of the RTC samples agree with the measured evaporation as well.

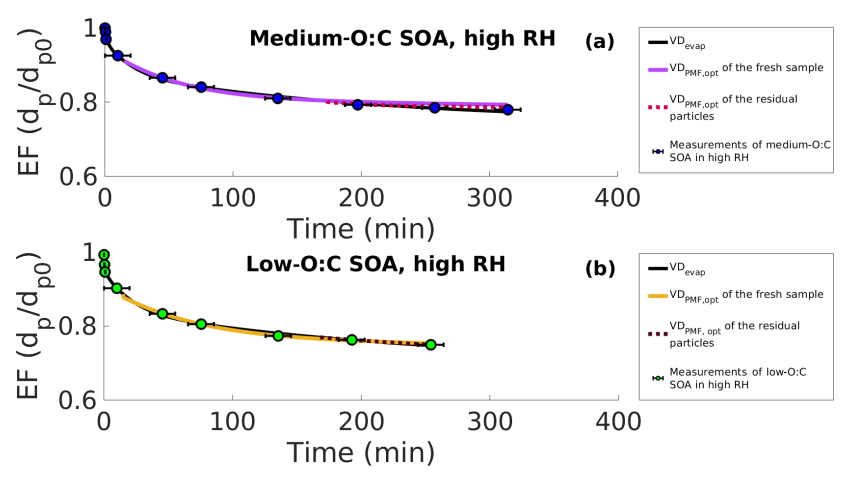

Figure 5. Evapograms of high-RH experiments showing the evaporation factors (circles), their uncertainty in time (black whiskers), the best-fit simulated evapogram calculated with $\mathrm{VD}_{\text {evap }}$ (solid black line) and the best-fit simulated evapograms calculated with the volatility distribution, where the effective saturation concentration $\left(\mathrm{C}^{*}\right)$ of each of the PMF factors is fitted to the measurements (VDPMF, opt). (a) Medium-O : C SOA. (b) Low-O : C SOA. The solid colored lines are for the fresh SOA, and the dashed lines are for the residual particles collected from the RTC after 173-259 and 168-254 min of evaporation for medium- and low-O : C SOAs, respectively. For fitting, the $\mathrm{C}^{*}$ of each PMF factor was allowed values from their respective 25 th and 75 th percentile desorption temperatures, as shown in Fig. 1. All the evapograms calculated with the VDPMF, opt match the measured evaporation, highlighting that the volatility distribution determined from the FIGAERO-CIMS data with the PMF method can describe the dynamics of evaporating SOA particles when uncertainties in the $\mathrm{C}^{*}$ of the factors are considered.

Overall, the results demonstrate that the information derived from the fresh and RTC FIGAERO-CIMS samples can 
describe the volatility of the evaporating particles when uncertainties in the desorption temperature are considered.

\subsection{Comparison of the volatility distribution of the fresh and RTC sample in high-RH conditions}

In this section, we compare VDPMF,opt of the fresh samples to VDPMF of the RTC sample to study if the two VD are similar. We compare the two VD at the mean evaporation time of the RTC sample. We calculated the evapograms with the VDPMF,opt of the fresh sample as the initial particle composition and recorded the mole fraction of each factor at the mean evaporation time of the RTC sample ( $216 \mathrm{~min}$ for medium-O : C particles and $211 \mathrm{~min}$ for low-O : C particles). Figure $6 \mathrm{a}$ and $\mathrm{c}$ show this comparison for both medium- and low-O : C particles. The factors are grouped into the three volatility classes described in Sect. 3.2. In Fig. 6, we show the results from the analysis where $\mathrm{VD}_{\mathrm{PMF}}$,opt was optimized by assigning the fresh sample composition at the mean sample evaporation time. Similar comparisons using the minimum and maximum evaporation time of the fresh sample are shown in Fig. S9 in the Supplement. To ensure that the factors are grouped to the same volatility classes for each studied VD, we used the $\mathrm{C}^{*}$ values of the $\mathrm{VD}_{\mathrm{PMF} \text {, opt }}$ at the mean sample evaporation time as a basis for the grouping.

The compositions simulated, based on the VDPMF,opt of the fresh samples, are comparable to the corresponding $\mathrm{VD}_{\mathrm{PMF}}$ of the RTC sample in both oxidation conditions (Fig. 6). The agreement is good, especially for the low-O : $\mathrm{C}$ case for which the $\mathrm{VD}_{\mathrm{PMF}}$,opt showed a slightly smaller contribution in volatility class 1 and a corresponding higher contribution in volatility class 2 compared to the VDPMF of the RTC sample (Fig. 6c). For the medium-O : C case, the VDPMF,opt predicted a higher contribution of volatility class 1 and a lower contribution of volatility class 2 compared to VDPMF (Fig. 6a).

These results show that the particle composition measured after a few hours of evaporation is consistent with the composition predicted, based on the composition observed at the start of evaporation, while considering the uncertainties of the interpreted $\mathrm{C}^{*}$ values.

\subsection{Volatility distribution comparison in dry conditions}

Next, we analyzed the evaporation experiments in dry conditions where the evaporation rate was reduced compared to the high-RH conditions. We interpreted this difference as an indication of particle-phase diffusion limitations in dry conditions (Yli-Juuti et al., 2017). Using the initial particle composition information obtained from the high-RH experiments and the FIGAERO-CIMS data, we explored the effect of particle viscosity on the evaporation process. Our aim is to test if the slower evaporation, presumably due to higher viscosity of the SOAs, can be captured with a recently developed viscosity parametrization based on glass transition tempera- tures of various organic compounds (DeRieux et al., 2018). We also compare the results, using the viscosity parametrization, to an approach where we fit both the viscosity and VD to the evapogram.

First, we investigated the range of particle viscosities that are required to explain the observed slower evaporation in dry conditions. For this, we simulated the particle evaporation in dry conditions based only on the evapogram data. We used the $\mathrm{VD}_{\text {evap }}$ (i.e., the initial particle composition obtained by optimizing mole fractions of VD bins with respect to the observed evapogram in high-RH conditions) as the initial particle composition estimate for the simulations and optimized the $b_{i}$ values (Eq. 3) for each VD bin. The best-fit simulation from this optimization agrees well with the observed size decrease in the dry experiments for both lowand medium-O : C particles (Fig. 8; black line). Based on these simulations, the viscosity of the particles needs to increase from below $10^{5} \mathrm{~Pa}$ s to approximately $10^{8} \mathrm{~Pa}$ during the evaporation in order to explain the evaporation rate observed for the dry particles.

Second, we tested the performance of the compositiondependent viscosity parameterization by DeRiuex et al. (2018) together with the PMF results. For this, we calculated the volatility distribution, VDPMF,dry, based on the $T_{\max }$ values of the factors from the fresh sample of the evaporation experiment at dry conditions (in the same way as for $\mathrm{VD}_{\mathrm{PMF}}$ for the high-RH case). The mole fraction of each factor was calculated from the mass loading profile to give the initial mole fraction of each VD bin for the simulations. We assigned this VDPMF,dry as the particle composition at the mean evaporation time of the fresh sample, i.e., $15 \mathrm{~min}$, and simulated the particle evaporation from there onwards. The particle size at the beginning of the simulation (i.e., at $15 \mathrm{~min}$ of evaporation) was taken from the above simulations and optimized based only on the evapogram data, which fitted well to the measurements. We calculated the viscosity parameter $b_{i}$ value for each VD bin, as described in Sect. 2.5, based on the mass spectra of the factor and the parameterization by DeRieux et al. (2018). In practice, this resulted in too high a viscosity for the particles to evaporate at all during the length of the experiment for both low- and medium-O : $\mathrm{C}$ particles (dashed gray line in Fig. 8). Therefore, we also conducted a simulation where the viscosity parameter $b_{i}$ value for each factor was calculated based on the viscosity parameterization by setting the $T_{\mathrm{g}}$ values of all compounds $30 \mathrm{~K}$ lower than the parametrization predicted, which is in line with the uncertainties reported by DeRiuex et al. (2018). In this case, the simulated evaporation was faster than observed for the medium- $\mathrm{O}: \mathrm{C}$ conditions (solid gray line in Fig. 8a) and similar to the evapogram calculated with the $\mathrm{VD}_{\text {evap }}$ for low-O : C conditions (solid gray line in Fig. 8b). This suggests that the observed evaporation rate at dry conditions and the viscosity parametrization by DeRieux et al. (2018) may be consistent with each other 

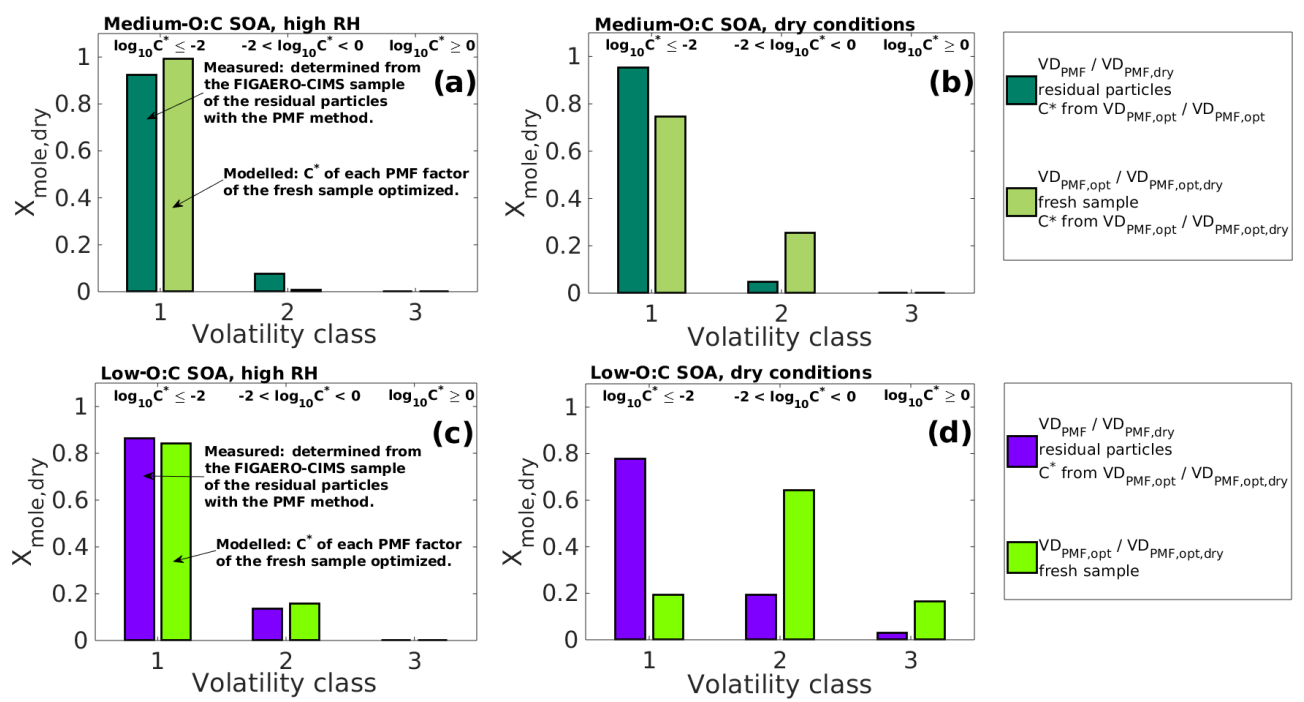

Figure 6. Comparison of the simulated particle composition (VDPMF,opt; VDPMF,opt,dry) to the particle composition determined from the residual particles collected from the RTC (VDPMF/VDPMF,dry) after 173-259 and 168-254 min of evaporation for medium- and low-O : C SOAs, respectively. The comparison is done at the mean evaporation time of the residual particles. The simulated compositions (VDPMF,opt $-\mathbf{a}$ and $\mathbf{c}$; VDPMF, opt,dry $-\mathbf{b}$ and $\mathbf{d}$ ) are taken from the best-fit simulated evapogram obtained from the optimization of the $\mathrm{C}^{*}$ values of the fresh sample's PMF factors to the measured evapogram. The volatility of the individual volatility distribution (VD) bins are grouped into three volatility classes, similar to Fig. 3. The limits for each volatility class are shown at the top and are the same for each subfigure. The $\mathrm{C}^{*}$ values from VDPMF, opt $/ \mathrm{VD}_{\mathrm{PMF}}$, opt, dry were used for corresponding $\mathrm{VD}_{\mathrm{PMF}} / \mathrm{VD}_{\mathrm{PMF}}$, dry when the volatility grouping was calculated in order to ensure the comparability. (a) Medium-O : C SOA in the high-RH experiment. (b) Medium-O : C SOA in the dry-condition experiment. (c) Low-O : C SOA in the high-RH experiment. (d) Low-O : C SOA in the dry-condition experiment. In the high-RH cases (a) and (c), the volatility distributions simulated, based on VDPMF, opt of the fresh SOAs, are similar to the measured VDPMF, while for the dry-condition cases (b) and (d), the volatility distributions simulated, based on VDPMF, opt,dry, show higher volatility than the measured VDPMF.

within the uncertainty range of the viscosity parametrization and the uncertainty range of the $\mathrm{C}^{*}$ of PMF factors.

Similar to Fig. 3, we show in Fig. 7 the comparison of VDPMF,dry $\left(\mathrm{C}^{*}\right.$ from $\left.T_{\max }\right)$ to the $\mathrm{VD}_{\text {evap }}$ in dry conditions and at the mean sample evaporation time, with the VD bins grouped into the three volatility classes. We show the mass loading profiles and the volatility distributions of the experiments in dry conditions in Figs. S10 and S11 in the Supplement. Figure S12 in the Supplement shows the same comparison as Fig. 7 for other sample evaporation times. For medium-O : C particles, VDPMF,dry calculated from the fresh sample has more contributions from volatility classes 1 and 3 and less from volatility class 2, compared to the corresponding $\mathrm{VD}_{\text {evap. }}$. For the low-O : C particles, the VDPMF,dry of the fresh sample has more contribution from volatility class 3 and less from volatility classes 1 and 2, compared to the $\mathrm{VD}_{\text {evap }}$. For medium-O : $\mathrm{C}$ particles, the differences between the VDPMF, dry and $\mathrm{VD}_{\text {evap }}$ leave open the possibility that the underestimated evaporation rate calculated using VDPMF, dry is partly a result of inaccuracy in the volatility description and not solely due to the high estimated viscosity. For the low-O : C particles, the underestimated evaporation most likely stems from the high estimated viscosity since VDPMF,dry is shifted towards higher volatility compounds than $\mathrm{VD}_{\text {evap. }}$.
As a third investigation of the viscosity, we again used the PMF results of the fresh sample in dry conditions to initialize the particle composition in the model at the mean fresh sample evaporation time. The mole fraction of each factor was calculated from the mass loading profile, giving the initial mole fraction of each VD bin for the simulations a similar one to the high-RH analysis. Then, using the MCGA algorithm together with the KM-GAP model, we estimated the $b_{i}$ coefficient and $\mathrm{C}^{*}$ of each VD bin by optimizing the KMGAP-simulated evapogram to the measured evapogram in dry conditions. This way, we obtained both the initial volatility distribution (VDPMF,dry,opt) and viscosity parameters $b_{i}$ simultaneously. For this optimization, we restricted the $\mathrm{C}^{*}$ values of the factors based on the 25th and 75th percentile of the desorption temperature of the factors (similar to what was done above for VDPMF,opt) and the viscosity parameter $b_{i}$ values, based on the DeRieux et al. (2018) parameterization. The $b_{i}$ values calculated with the original parametrization by DeRieux et al. (2018) were set as the upper limit for the $b_{i}$ values. The lower limit for the $b_{i}$ values was calculated by setting the glass transition temperature of each compound $30 \mathrm{~K}$ lower than the parametrization predicted. As above, in these simulations the initial particle size was also taken from the simulations where the optimization was based only on the evapogram data. For both medium- and low-O : C particles, 

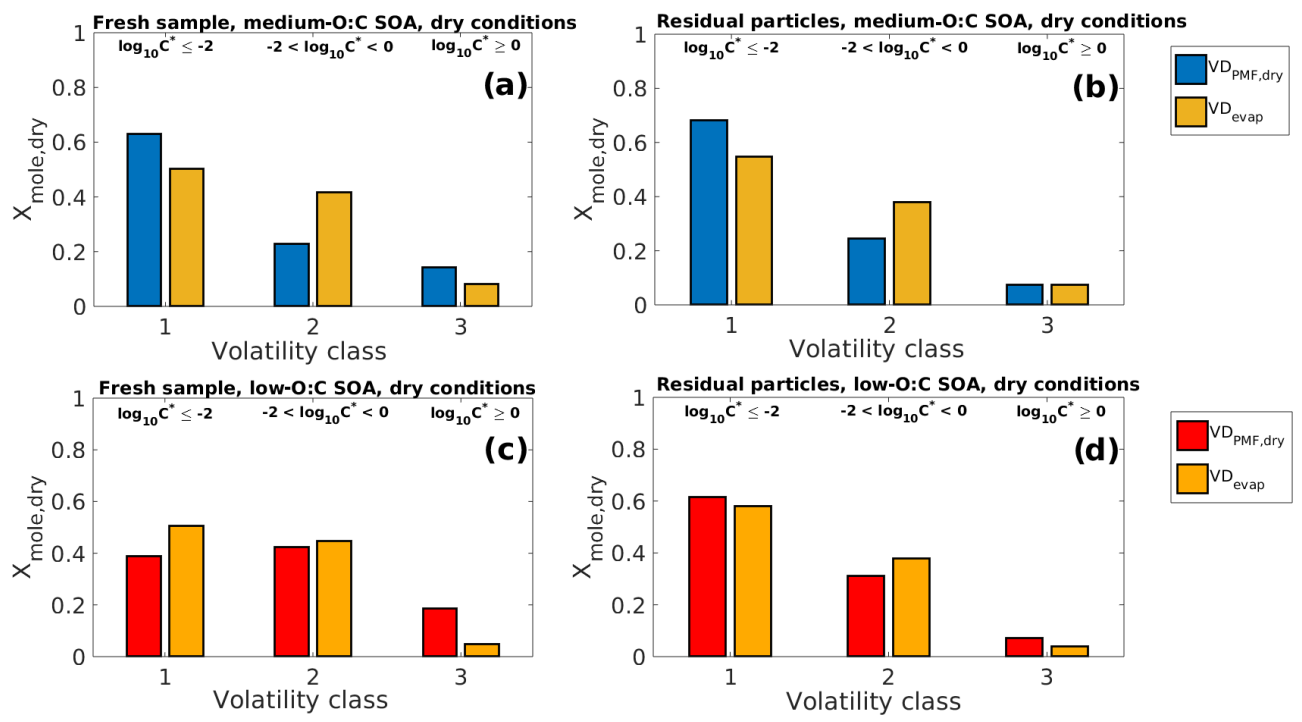

Figure 7. Comparison of $\mathrm{VD}_{\mathrm{PMF}}$, dry (volatility distribution with $\mathrm{C}^{*}$ calculated from the peak desorption temperature, $T_{\mathrm{max}}$, of each PMF factor) and $\mathrm{VD}_{\text {evap }}$ (volatility distribution determined by fitting the LLEVAP model to the measured evapogram) at the mean evaporation time of the SOA samples in dry-condition experiments. The VD bins are grouped into three volatility classes, similar to Fig. 3 . The limits for each volatility class are shown at the top and are the same for each subfigure. (a) Fresh sample of medium-O : C SOA. (b) Residual particles of medium-O : C SOA after 170-256 min of evaporation (the RTC sample). (c) Fresh sample of low-O : C SOA. (d) Residual particles of low-O : C SOA after 152-238 min of evaporation (the RTC sample). The VDPMF, dry shows lower overall volatility than the VDevap for medium-O : C SOA. For low-O : C SOA, the VDPMF, dry shows higher volatility for the fresh sample and similar volatility compared to the VDevap after 152-238 min of evaporation.
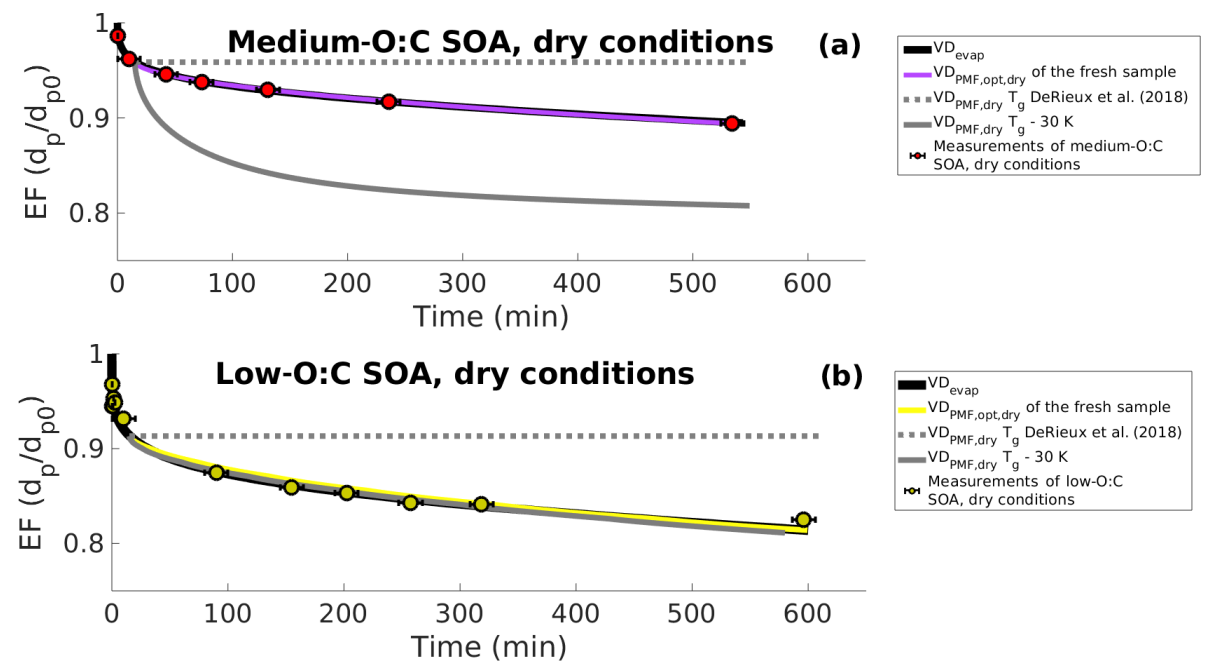

Figure 8. Evapograms showing the measured isothermal evaporation of (a) medium-O : C SOA and (b) low-O : C SOA in dry-condition experiments (markers and black whiskers) together with the simulated evapograms. The best-fit simulated evapogram calculated with $\mathrm{VD}_{\text {evap }}$ (obtained from high-RH experiments) and optimizing $b_{i}$ is shown with a solid black line. Gray lines show the minimum and maximum possible evaporation calculated, with the VDPMF, dry $\left(\mathrm{C}^{*}\right.$ of PMF factors calculated from $\left.T_{\max }\right)$ at the highest (the original parametrization of DeRieux et al., 2018; dashed gray lines) or the lowest (30 K subtracted from the $T_{\mathrm{g}}$ of every ion; solid gray line) studied viscosity. Solid purple and yellow lines show the best-fit simulated evapograms calculated with the optimized VDPMF,opt,dry (based on the assumption that the FIGAERO sample represents particles at the mean of the sample collection interval) and $b_{i}$ restricted (based on the DeRieux et al., 2018, parameterization). The figure shows, similar to Fig. 5, that the volatility distribution determined from the FIGAERO-CIMS data with the PMF method is consistent with the measured evaporation of the SOA particles once the uncertainty in the effective saturation concentration and the glass transition temperature parametrization of DeRieux et al. (2018) are considered. 
it was possible to find a set of $\mathrm{C}^{*}$ and $b_{i}$ values that produced an equally good match to the experimental data as the $\mathrm{VD}_{\text {evap }}$ (purple and yellow lines in Fig. 8).

Figure $6 \mathrm{~b}$ and $\mathrm{d}$ show the comparison of the measured and simulated particle composition, grouped into the three volatility classes, at the RTC sample collection time for the dry experiments for low- and medium-O : C particles. The measured composition is the VD calculated from the PMF results of the RTC samples in dry conditions. The optimized $\mathrm{C}^{*}$ values of the factors from the corresponding dry experiment were used for these VD. The simulated particle composition is taken from the optimized model run (optimized VDPMF,opt,dry and $b_{i}$ ) at the mean RTC sample collection time, similar to the high-RH cases presented in Fig. 6a and c. For low-O : C particles, there is a clear discrepancy as the VDPMF,dry,opt implies a much larger relative contribution from volatility classes 2 and 3 and a smaller contribution from volatility class 1 when compared to the measurements. This inconsistency may be related to the rather high viscosities in the simulations. The viscosity of the low$\mathrm{O}: \mathrm{C}$ particles in this optimized simulation was rather high, at $\eta>10^{8} \mathrm{~Pa}$ s throughout the evaporation, slowing the evaporation of the higher volatility compounds. A similar evaporation curve could be obtained with lower viscosity and lower volatilities of the VD bins.

\section{Discussion}

Qualitatively, VD $D_{\mathrm{PMF}}$ and $\mathrm{VD}_{\mathrm{PMF}}$,dry capture the evaporation dynamics well in all studied cases; quantitatively, there were discrepancies. For the VDPMF of the fresh samples, the first and second factor desorb at low heating temperatures (below $100^{\circ} \mathrm{C}$ ), indicating that these factors represent high-volatility organic compounds that evaporate almost completely from the particles in the experimental timescale of our isothermal evaporation experiments. In the RTC samples, these factors show significantly lower or nonexisting signal strength relative to the other factors. The factors that desorb at high temperatures show an increase in the relative signal strength in the RTC samples compared to the fresh samples, which is consistent with the expected increase in the relative contribution of lower volatility compounds along evaporation. These findings indicate that the FIGAERO-CIMS measurements of $\alpha$-pinene SOAs and the applied PMF method give a good overall picture of the evolution of the volatility distribution during evaporation.

In addition to the PMF method used here, other ways of characterizing SOA compound volatilities or VBS from FIGAERO-CIMS thermograms have also been suggested (e.g., Stark et al., 2017). These include, for example, the more straightforward method of calculating the $\mathrm{C}^{*}$ of each detected ion based on their $T_{\max }$, using Eq. (3) and lumping them into a traditional VBS. While such other methods may capture the volatility distributions sufficiently, the benefit of
PMF method is that it offers a new way to understand what happens inside the particles, e.g., during the heating in FIGAERO. Here we have evaluated this method with respect to its ability to capture the volatilities of SOAs.

At high RH, the VDPMF that was derived from the $T_{\max }$ of each factor's mass loading profile did not produce an evapogram similar to the measured ones when the VDPMF was used as an input for the LLEVAP model. This reflects the sensitivity of the particle evaporation to the $\mathrm{C}^{*}$ values and suggests that the VDPMF is not directly applicable as a particle composition estimate for a detailed particle dynamics study. When we allowed uncertainty in the $\mathrm{C}^{*}$ values of each factor, we were able to explain most of the discrepancies between the simulated and measured evapograms. Our results also demonstrate the need for careful investigation of the representative time of the sample when filter-collected samples are applied for dynamic processes such as evaporation.

In this study we assumed a quite large uncertainty range for the desorption temperature of each PMF factor, and it is not certain that the determination of $\mathrm{VD}_{\mathrm{PMF}}$,opt would be successful if the allowed ranges for $\mathrm{C}^{*}$ of PMF factors were lower. Thus, work remains to be done in studying what the total uncertainty is that arises from combining the FIGAEROCIMS measurements with the PMF method and to what extent the PMF factors can be thought to represent surrogate organic compounds for the purpose of detailed SOA dynamics studies.

We note that care has to be taken when PMF results are transferred to volatility distributions, especially with regard to separating the contribution of instrument background and contamination from the true sample. When the sample mass was low (in the low-O : C RTC sample), we noticed that the first half of the bimodal (factor LD1a) resulted in a high mole fraction even though the absolute signal strength of the factor did not change between the fresh and the RTC sample, which is usually an indication that this signal is caused by instrument background. However, the signal strength of this factor was low enough in all cases to not affect the overall VD estimation. More details on the interpretation of B- and D-type factors and potential factor blending can be found in Buchholz et al (2020).

In dry conditions, VDPMF, dry of the fresh sample in the low-O : C case showed a noticeably higher amount of highvolatility matter than $\mathrm{VD}_{\text {evap }}$. This discrepancy between the volatility distributions is not expected and raises a need for further studies on the role of viscosity and possible particlephase chemistry in SOA particle dynamics. Future studies should investigate the possibility of chemical reactions that modify the volatility of organic compounds and how viscosity is described in process models. 


\section{Conclusions}

We compared volatility distributions derived from FIGAERO-CIMS measurements with PMF analysis to volatility distributions derived from fitting a process model to match the measured size change in particles during isothermal evaporation. We compared the two methods for obtaining the volatility-distribution data for two different particle compositions and two evaporation conditions. The results are promising and suggest that the methods provide volatility distributions that are in agreement. We note that the data set available here is limited and additional investigations on comparing the methods are desirable in the future.

In all studied experimental data sets, we were able to capture the measured evaporation with the fitting method. In high-RH experiments, VDPMF deviated from $\mathrm{VD}_{\text {evap, }}$, especially when the FIGAERO samples were collected at the early stages of the evaporation. However, qualitatively, both types of VD evolved similarly, i.e., the fraction of lower volatility compounds increased, and the fraction of higher volatility compounds decreased during the evaporation of the particles. These results suggest that the changes in FIGAERO-CIMS-derived volatility distributions over the isothermal evaporation are consistent with the observed isothermal evaporation, and the detailed SOA dynamics are sensitive to the uncertainties in the $\mathrm{C}^{*}$ values.

The volatility distribution derived with the PMF method at high RH agreed with the observed isothermal evaporation better when we interpreted the volatility of each factor as a range of possible $\mathrm{C}^{*}$ values and optimized the $\mathrm{C}^{*}$ values within these ranges with respect to the measurements. These results suggest that the FIGAERO-CIMS measurements combined with PMF method not only provide qualitative information of the volatilities of the SOA constituents but they also have the potential for quantitative investigations of the volatility distributions. However, more work is needed to constrain the uncertainties rising from the conversion of the FIGAERO-CIMS desorption temperatures to $\mathrm{C}^{*}$ values, and it should be noted that deriving the volatilities based on only the $T_{\max }$ of PMF factors may not be sufficient for representing detailed SOA dynamics.

In dry conditions, we were able to simulate the evapograms based on the PMF results, using the VTF equation and the glass transition temperature parametrization of DeRieux et al. (2018), if both $C^{*}$ and viscosity parameters were optimized and allowed to contain reasonable uncertainties. For both oxidation conditions, the measured composition at the later stages of the evaporation suggested considerably lower volatility than the simulations. These results suggest that the tested viscosity parameterization is not in disagreement with the observed SOA evaporation; however, the uncertainties related to the method are significant from the point of view of simulating SOA dynamics.

Based on our analysis, we conclude that using the PMF method with FIGAERO-CIMS thermogram data is good for estimating the volatility distribution of organic aerosols when the organic compounds present in the particle phase have low volatilities with respect to the sample collection and analysis timescale. Specifically, VDPMF is useful for extracting information about organic compounds that do not evaporate during the evaporation measurements at room temperature. VDPMF is applicable for detailed particle dynamics studies when the desorption temperature of the factor is characterized with a range around the $T_{\max }$ value. Furthermore, combining VDPMF,opt with detailed process modeling and input optimization could allow the quantification of other physical or chemical properties of organic aerosols since the FIGAERO-CIMS data constrain the particle composition and effectively decrease the search space that needs to be explored with global optimization methods.

Code availability. The process models used in this study can be acquired on request from the corresponding author. The MCGA code is available at https://doi.org/10.5281/zenodo.3759733 (Tikkanen, 2020).

Supplement. The supplement related to this article is available online at: https://doi.org/10.5194/acp-20-10441-2020-supplement.

Author contributions. OPT, AB, SS, AV and TYJ designed the study. OPT did the calculations, with support from AB and TYJ, except for the PMF calculations which were done by AB. AY developed the calibration method for calculating $\mathrm{C}^{*}$ from the desorption temperature, with support from SS. All authors participated in the interpretation of the data. OPT wrote the paper with contributions from all coauthors.

Competing interests. The authors declare that they have no conflict of interest.

Acknowledgements. The authors would like to thank Claudia Mohr and Wei Huang for the use of the FIGAERO instrument from the Karlsruhe Institute of Technology and their support during the FIGAERO-CIMS data analysis. Furthermore, we want to acknowledge Andrew Lambe and Aerodyne Research, Inc. for lending us a potential aerosol mass reactor.

Financial support. This work has been supported by the Academy of Finland Center of Excellence program (grant no. 307331), the Academy of Finland (grant nos. 299544 and 310682), European Research Council (ERC StG QAPPA; grant no. 335478) and the University of Eastern Finland Doctoral Programme in Environmental Physics, Health and Biology. 
Review statement. This paper was edited by Neil M. Donahue and reviewed by Neil M. Donahue and three anonymous referees.

\section{References}

Angell, C. A.: Formation of glasses from liquids and biopolymers, Science, 267, 1924-1935, 1995.

Angell, C. A.: Liquid Fragility and the Glass Transition in Water and Aqueous Solutions, Chem. Rev., 102, 2627-2650, https://doi.org/10.1021/cr000689q, 2002.

Bannan, T. J., Le Breton, M., Priestley, M., Worrall, S. D., Bacak, A., Marsden, N. A., Mehra, A., Hammes, J., Hallquist, M., Alfarra, M. R., Krieger, U. K., Reid, J. P., Jayne, J., Robinson, W., McFiggans, G., Coe, H., Percival, C. J., and Topping, D.: A method for extracting calibrated volatility information from the FIGAERO-HR-ToF-CIMS and its experimental application, Atmos. Meas. Tech., 12, 1429-1439, https://doi.org/10.5194/amt12-1429-2019, 2019.

Berkemeier, T., Ammann, M., Krieger, U. K., Peter, T., Spichtinger, P., Pöschl, U., Shiraiwa, M., and Huisman, A. J.: Technical note: Monte Carlo genetic algorithm (MCGA) for model analysis of multiphase chemical kinetics to determine transport and reaction rate coefficients using multiple experimental data sets, Atmos. Chem. Phys., 17, 8021-8029, https://doi.org/10.5194/acp17-8021-2017, 2017.

Buchholz, A., Lambe, A. T., Ylisirniö, A., Li, Z., Tikkanen, O.P., Faiola, C., Kari, E., Hao, L., Luoma, O., Huang, W., Mohr, C., Worsnop, D. R., Nizkorodov, S. A., Yli-Juuti, T., Schobesberger, S., and Virtanen, A.: Insights into the $\mathrm{O}: \mathrm{C}$-dependent mechanisms controlling the evaporation of $\alpha$-pinene secondary organic aerosol particles, Atmos. Chem. Phys., 19, 4061-4073, https://doi.org/10.5194/acp-19-4061-2019, 2019.

Buchholz, A., Ylisirniö, A., Huang, W., Mohr, C., Canagaratna, M., Worsnop, D. R., Schobesberger, S., and Virtanen, A.: Deconvolution of FIGAERO-CIMS thermal desorption profiles using positive matrix factorisation to identify chemical and physical processes during particle evaporation, Atmos. Chem. Phys., 20, 7693-7716, https://doi.org/10.5194/acp-20-7693-2020, 2020.

Canagaratna, M. R., Jayne, J. T., Jimenez, J. L., Allan, J. D., Alfarra, M. R., Zhang, Q., Onasch, T. B., Drewnick, F., Coe, H., Middlebrook, A., Delia, A., Williams, L. R., Trimborn, A. M., Northway, M. J., DeCarlo, P. F., Kolb, C. E., Davidovits, P., and Worsnop, D. R.: Chemical and microphysical characterization of ambient aerosols with the aerodyne aerosol mass spectrometer, Mass Spectrom. Rev., 26, 185-222, https://doi.org/10.1002/mas.20115, 2007.

D’Ambro, E. L., Lee, B. H., Liu, J., Shilling, J. E., Gaston, C. J., Lopez-Hilfiker, F. D., Schobesberger, S., Zaveri, R. A., Mohr, C., Lutz, A., Zhang, Z., Gold, A., Surratt, J. D., Rivera-Rios, J. C., Keutsch, F. N., and Thornton, J. A.: Molecular composition and volatility of isoprene photochemical oxidation secondary organic aerosol under low- and high- $\mathrm{NO}_{x}$ conditions, Atmos. Chem. Phys., 17, 159-174, https://doi.org/10.5194/acp-17159-2017, 2017.

D’Ambro, E. L., Schobesberger, S., Zaveri, R. A., Shilling, J. E., Lee, B. H., Lopez-Hilfiker, F. D., Mohr, C., and Thornton, J. A.: Isothermal Evaporation of $\alpha$-Pinene Ozonolysis SOA: Volatility, Phase State, and Oligomeric
Composition, ACS Earth Space Chem., 2, 1058-1067, https://doi.org/10.1021/acsearthspacechem.8b00084, 2018.

DeCarlo, P. F., Kimmel, J. R., Trimborn, A., Northway, M. J., Jayne, J. T., Aiken, A. C., Gonin, M., Fuhrer, K., Horvath, T., Docherty, K. S., Worsnop, D. R., and Jimenez, J. L.: Field-Deployable, High-Resolution, Time-ofFlight Aerosol Mass Spectrometer, Anal. Chem., 78, 8281-8289, https://doi.org/10.1021/ac061249n, 2006.

DeRieux, W.-S. W., Li, Y., Lin, P., Laskin, J., Laskin, A., Bertram, A. K., Nizkorodov, S. A., and Shiraiwa, M.: Predicting the glass transition temperature and viscosity of secondary organic material using molecular composition, Atmos. Chem. Phys., 18, 6331-6351, https://doi.org/10.5194/acp-18-6331-2018, 2018.

Dette, H. P., Qi, M., Schröder, D. C., Godt, A., and Koop, T.: GlassForming Properties of 3-Methylbutane-1,2,3-tricarboxylic Acid and Its Mixtures with Water and Pinonic Acid, J. Phys. Chem. A, 118, 7024-7033, https://doi.org/10.1021/jp505910w, 2014.

Donahue, N. M., Robinson, A. L., Stanier, C. O., and Pandis, S. N.: Coupled partitioning, dilution, and chemical aging of semivolatile organics, Environ. Sci. Technol., 40, 2635-2643, https://doi.org/10.1021/es052297c, 2006.

Gedeon, O.: Origin of glass fragility and Vogel temperature emerging from Molecular dynamics simulations, J. Non-Cryst. Solids, 498, 109-117, https://doi.org/10.1016/j.jnoncrysol.2018.06.012, 2018.

Glasius, M. and Goldstein, A. H.: Recent Discoveries and Future Challenges in Atmospheric Organic Chemistry, Environ. Sci. Technol., 50, 2754-2764, https://doi.org/10.1021/acs.est.5b05105, 2016.

Goldstein, A. H. and Galbally, I. E.: Known and Unexplored Organic Constituents in the Earth's Atmosphere, Environ. Sci. Technol., 41, 1514-1521, https://doi.org/10.1021/es072476p, 2007.

Hallquist, M., Wenger, J. C., Baltensperger, U., Rudich, Y., Simpson, D., Claeys, M., Dommen, J., Donahue, N. M., George, C., Goldstein, A. H., Hamilton, J. F., Herrmann, H., Hoffmann, T., Iinuma, Y., Jang, M., Jenkin, M. E., Jimenez, J. L., Kiendler-Scharr, A., Maenhaut, W., McFiggans, G., Mentel, Th. F., Monod, A., Prévôt, A. S. H., Seinfeld, J. H., Surratt, J. D., Szmigielski, R., and Wildt, J.: The formation, properties and impact of secondary organic aerosol: current and emerging issues, Atmos. Chem. Phys., 9, 5155-5236, https://doi.org/10.5194/acp9-5155-2009, 2009.

Huang, W., Saathoff, H., Pajunoja, A., Shen, X., Naumann, K.H., Wagner, R., Virtanen, A., Leisner, T., and Mohr, C.: $\alpha$ Pinene secondary organic aerosol at low temperature: chemical composition and implications for particle viscosity, Atmos. Chem. Phys., 18, 2883-2898, https://doi.org/10.5194/acp18-2883-2018, 2018.

Isaacman-VanWertz, G., Massoli, P., E. O’Brien, R., B. Nowak, J., R. Canagaratna, M., T. Jayne, J., R. Worsnop, D., Su, L., A. Knopf, D., K. Misztal, P., Arata, C., H. Goldstein, A., and H. Kroll, J.: Using advanced mass spectrometry techniques to fully characterize atmospheric organic carbon: current capabilities and remaining gaps, Faraday Discuss., 200, 579-598, https://doi.org/10.1039/C7FD00021A, 2017.

Isaacman-VanWertz, G., Massoli, P., O’Brien, R., Lim, C., Franklin, J. P., Moss, J. A., Hunter, J. F., Nowak, J. B., Canagaratna, M. R., Misztal, P. K., Arata, C., Roscioli, J. R., Herndon, S. T., Onasch, 
T. B., Lambe, A. T., Jayne, J. T., Su, L., Knopf, D. A., Goldstein, A. H., Worsnop, D. R., and Kroll, J. H.: Chemical evolution of atmospheric organic carbon over multiple generations of oxidation, Nat. Chem., 10, 462-468, https://doi.org/10.1038/s41557018-0002-2, 2018.

Iyer, S., Lopez-Hilfiker, F., Lee, B. H., Thornton, J. A., and Kurtén, T.: Modeling the Detection of Organic and Inorganic Compounds Using Iodide-Based Chemical Ionization, J. Phys. Chem. A, 120, 576-587, https://doi.org/10.1021/acs.jpca.5b09837, 2016.

Jayne, J. T., Leard, D. C., Zhang, X., Davidovits, P., Smith, K. A., Kolb, C. E., and Worsnop, D. R.: Development of an Aerosol Mass Spectrometer for Size and Composition Analysis of Submicron Particles, Aerosol Sci. Technol., 33, 49-70, https://doi.org/10.1080/027868200410840, 2000.

Jimenez, J. L., Canagaratna, M. R., Donahue, N. M., Prevot, A. S. H., Zhang, Q., Kroll, J. H., DeCarlo, P. F., Allan, J. D., Coe, H., Ng, N. L., Aiken, A. C., Docherty, K. S., Ulbrich, I. M., Grieshop, A. P., Robinson, A. L., Duplissy, J., Smith, J. D., Wilson, K. R., Lanz, V. A., Hueglin, C., Sun, Y. L., Tian, J., Laaksonen, A., Raatikainen, T., Rautiainen, J., Vaattovaara, P., Ehn, M., Kulmala, M., Tomlinson, J. M., Collins, D. R., Cubison, M. J., Dunlea, E. J., Huffman, J. A., Onasch, T. B., Alfarra, M. R., Williams, P. I., Bower, K., Kondo, Y., Schneider, J., Drewnick, F., Borrmann, S., Weimer, S., Demerjian, K., Salcedo, D., Cottrell, L., Griffin, R., Takami, A., Miyoshi, T., Hatakeyama, S., Shimono, A., Sun, J. Y., Zhang, Y. M., Dzepina, K., Kimmel, J. R., Sueper, D., Jayne, J. T., Herndon, S. C., Trimborn, A. M., Williams, L. R., Wood, E. C., Middlebrook, A. M., Kolb, C. E., Baltensperger, U., Worsnop, D. R., and Worsnop, D. R.: Evolution of organic aerosols in the atmosphere., Science, 326, 15259, https://doi.org/10.1126/science.1180353, 2009.

Kang, E., Root, M. J., Toohey, D. W., and Brune, W. H.: Introducing the concept of Potential Aerosol Mass (PAM), Atmos. Chem. Phys., 7, 5727-5744, https://doi.org/10.5194/acp-7-5727-2007, 2007.

Lambe, A. T., Ahern, A. T., Williams, L. R., Slowik, J. G., Wong, J. P. S., Abbatt, J. P. D., Brune, W. H., Ng, N. L., Wright, J. P., Croasdale, D. R., Worsnop, D. R., Davidovits, P., and Onasch, T. B.: Characterization of aerosol photooxidation flow reactors: heterogeneous oxidation, secondary organic aerosol formation and cloud condensation nuclei activity measurements, Atmos. Meas. Tech., 4, 445-461, https://doi.org/10.5194/amt-4445-2011, 2011.

Le Breton, M., Wang, Y., Hallquist, Å. M., Pathak, R. K., Zheng, J., Yang, Y., Shang, D., Glasius, M., Bannan, T. J., Liu, Q., Chan, C. K., Percival, C. J., Zhu, W., Lou, S., Topping, D., Wang, Y., Yu, J., Lu, K., Guo, S., Hu, M., and Hallquist, M.: Online gasand particle-phase measurements of organosulfates, organosulfonates and nitrooxy organosulfates in Beijing utilizing a FIGAERO ToF-CIMS, Atmos. Chem. Phys., 18, 10355-10371, https://doi.org/10.5194/acp-18-10355-2018, 2018.

Lee, B. H., Lopez-Hilfiker, F. D., Mohr, C., Kurtén, T., Worsnop, D. R., and Thornton, J. A.: An Iodide-Adduct HighResolution Time-of-Flight Chemical-Ionization Mass Spectrometer: Application to Atmospheric Inorganic and Organic Compounds, Environ. Sci. Technol., 48, 6309-6317, https://doi.org/10.1021/es500362a, 2014.

Lee, B. H., Lopez-Hilfiker, F. D., D’Ambro, E. L., Zhou, P., Boy, M., Petäjä, T., Hao, L., Virtanen, A., and Thornton, J. A.:
Semi-volatile and highly oxygenated gaseous and particulate organic compounds observed above a boreal forest canopy, Atmos. Chem. Phys., 18, 11547-11562, https://doi.org/10.5194/acp-1811547-2018, 2018.

Lehtinen, K. E. J. and Kulmala, M.: A model for particle formation and growth in the atmosphere with molecular resolution in size, Atmos. Chem. Phys., 3, 251-257, https://doi.org/10.5194/acp-3251-2003, 2003.

Lopez-Hilfiker, F. D., Mohr, C., Ehn, M., Rubach, F., Kleist, E., Wildt, J., Mentel, Th. F., Lutz, A., Hallquist, M., Worsnop, D., and Thornton, J. A.: A novel method for online analysis of gas and particle composition: description and evaluation of a Filter Inlet for Gases and AEROsols (FIGAERO), Atmos. Meas. Tech., 7, 983-1001, https://doi.org/10.5194/amt-7-983-2014, 2014.

Lopez-Hilfiker, F. D., Mohr, C., Ehn, M., Rubach, F., Kleist, E., Wildt, J., Mentel, Th. F., Carrasquillo, A. J., Daumit, K. E., Hunter, J. F., Kroll, J. H., Worsnop, D. R., and Thornton, J. A.: Phase partitioning and volatility of secondary organic aerosol components formed from $\alpha$-pinene ozonolysis and $\mathrm{OH}$ oxidation: the importance of accretion products and other low volatility compounds, Atmos. Chem. Phys., 15, 7765-7776, https://doi.org/10.5194/acp-15-7765-2015, 2015.

Lopez-Hilfiker, F. D., Mohr, C., D’Ambro, E. L., Lutz, A., Riedel, T. P., Gaston, C. J., Iyer, S., Zhang, Z., Gold, A., Surratt, J. D., Lee, B. H., Kurten, T., Hu, W. W., Jimenez, J., Hallquist, M., and Thornton, J. A.: Molecular Composition and Volatility of Organic Aerosol in the Southeastern U.S.: Implications for IEPOX Derived SOA, Environ. Sci. Technol., 50, 2200-2209, https://doi.org/10.1021/acs.est.5b04769, 2016.

Paatero, P. and Tapper, U.: Positive matrix factorization: A non-negative factor model with optimal utilization of error estimates of data values, Environmetrics, 5, 111-126, https://doi.org/10.1002/env.3170050203, 1994.

Pathak, R. K., Presto, A. A., Lane, T. E., Stanier, C. O., Donahue, N. M., and Pandis, S. N.: Ozonolysis of $\alpha$-pinene: parameterization of secondary organic aerosol mass fraction, Atmos. Chem. Phys., 7, 3811-3821, https://doi.org/10.5194/acp-7-3811-2007, 2007.

Reid, J. P., Bertram, A. K., Topping, D. O., Laskin, A., Martin, S. T., Petters, M. D., Pope, F. D., and Rovelli, G.: The viscosity of atmospherically relevant organic particles, Nat. Commun., 9, 956, https://doi.org/10.1038/s41467-018-03027-z, 2018.

Reid, R. C., Prausnitz, J. M., and Poling, B. E.: The properties of gases and liquids, 4th Edn., McGraw Hill Book Co, New York,NY, USA, 1987.

Renbaum-Wolff, L., Grayson, J. W., Bateman, A. P., Kuwata, M., Sellier, M., Murray, B. J., Shilling, J. E., Martin, S. T., and Bertram, A. K.: Viscosity of $\alpha$-pinene secondary organic material and implications for particle growth and reactivity, P. Natl. Acad. Sci. USA, 110, 8014-8019, https://doi.org/10.1073/pnas.1219548110, 2013.

Schobesberger, S., D’Ambro, E. L., Lopez-Hilfiker, F. D., Mohr, C., and Thornton, J. A.: A model framework to retrieve thermodynamic and kinetic properties of organic aerosol from composition-resolved thermal desorption measurements, Atmos. Chem. Phys., 18, 14757-14785, https://doi.org/10.5194/acp-1814757-2018, 2018.

Shiraiwa, M., Pfrang, C., Koop, T., and Pöschl, U.: Kinetic multilayer model of gas-particle interactions in aerosols and clouds (KM-GAP): linking condensation, evaporation and chemical re- 
actions of organics, oxidants and water, Atmos. Chem. Phys., 12, 2777-2794, https://doi.org/10.5194/acp-12-2777-2012, 2012.

Shiraiwa, M., Li, Y., Tsimpidi, A. P., Karydis, V. A., Berkemeier, T., Pandis, S. N., Lelieveld, J., Koop, T., and Pöschl, U.: Global distribution of particle phase state in atmospheric secondary organic aerosols, Nat. Commun., 8, 15002, https://doi.org/10.1038/ncomms15002, 2017.

Stark, H., Yatavelli, R. L. N., Thompson, S. L., Kang, H., Krechmer, J. E., Kimmel, J. R., Palm, B. B., Hu, W., Hayes, P. L., Day, D. A., Campuzano-Jost, P., Canagaratna, M. R., Jayne, J. T., Worsnop, D. R., and Jimenez, J. L.: Impact of Thermal Decomposition on Thermal Desorption Instruments: Advantage of Thermogram Analysis for Quantifying Volatility Distributions of Organic Species, Environ. Sci. Technol., 51, 8491-8500, https://doi.org/10.1021/acs.est.7b00160, 2017.

Stocker, T. F., Qin, D., Plattner, G.-K., Tignor, M., Allen, S. K., Boschung, J., Nauels, A., Xia, Y., Bex, V., Midgley, P. M., and others: Climate change 2013: The physical science basis, Cambridge University Press, Cambridge, 2013.

Tikkanen, O.-P.: Altesmi/uMCGA_matlab: First version (Version v1.0.0), Zenodo, https://doi.org/10.5281/zenodo.3759733, 2020.

Tikkanen, O.-P., Hämäläinen, V., Rovelli, G., Lipponen, A., Shiraiwa, M., Reid, J. P., Lehtinen, K. E. J., and Yli-Juuti, T.: Optimization of process models for determining volatility distribution and viscosity of organic aerosols from isothermal particle evaporation data, Atmos. Chem. Phys., 19, 9333-9350, https://doi.org/10.5194/acp-19-9333-2019, 2019.

Vaden, T. D., Imre, D., Beránek, J., Shrivastava, M., and Zelenyuk, A.: Evaporation kinetics and phase of laboratory and ambient secondary organic aerosol., P. Natl. Acad. Sci. USA., 108, 21902195, https://doi.org/10.1073/pnas.1013391108, 2011.

Vesala, T., Kulmala, M., Rudolf, R., Vrtala, A., and Wagner, P. E.: Models for condensational growth and evaporation of binary aerosol particles, J. Aerosol Sci., 28, 565-598, https://doi.org/10.1016/S0021-8502(96)00461-2, 1997.
Virtanen, A., Joutsensaari, J., Koop, T., Kannosto, J., Yli-Pirilä, P., Leskinen, J., Mäkelä, J. M., Holopainen, J. K., Pöschl, U., Kulmala, M., Worsnop, D. R., and Laaksonen, A.: An amorphous solid state of biogenic secondary organic aerosol particles, Nature, 467, 824-827, https://doi.org/10.1038/nature09455, 2010.

Wilson, J., Imre, D., Beraek, J., Shrivastava, M., and Zelenyuk, A.: Evaporation Kinetics of Laboratory-Generated Secondary Organic Aerosols at Elevated Relative Humidity, Environ. Sci. Technol., 49, 243-249, https://doi.org/10.1021/es505331d, 2015.

Yli-Juuti, T., Pajunoja, A., Tikkanen, O.-P., Buchholz, A., Faiola, C., Väisänen, O., Hao, L., Kari, E., Peräkylä, O., Garmash, O., Shiraiwa, M., Ehn, M., Lehtinen, K., and Virtanen, A.: Factors controlling the evaporation of secondary organic aerosol from $\alpha$-pinene ozonolysis, Geophys. Res. Lett., 44, 2562-2570, https://doi.org/10.1002/2016GL072364, 2017.

Zhang, Q., Alfarra, M. R., Worsnop, D. R., Allan, J. D., Coe, H., Canagaratna, M. R., and Jimenez, J. L.: Deconvolution and Quantification of Hydrocarbon-like and Oxygenated Organic Aerosols Based on Aerosol Mass Spectrometry, Environ. Sci. Technol., 39, 4938-4952, https://doi.org/10.1021/es0485681, 2005.

Zhang, Q., Jimenez, J. L., Canagaratna, M. R., Allan, J. D., Coe, H., Ulbrich, I., Alfarra, M. R., Takami, A., Middlebrook, A. M., Sun, Y. L., Dzepina, K., Dunlea, E., Docherty, K., DeCarlo, P. F., Salcedo, D., Onasch, T., Jayne, J. T., Miyoshi, T., Shimono, A., Hatakeyama, S., Takegawa, N., Kondo, Y., Schneider, J., Drewnick, F., Borrmann, S., Weimer, S., Demerjian, K., Williams, P., Bower, K., Bahreini, R., Cottrell, L., Griffin, R. J., Rautiainen, J., Sun, J. Y., Zhang, Y. M., and Worsnop, D. R.: Ubiquity and dominance of oxygenated species in organic aerosols in anthropogenically-influenced Northern Hemisphere midlatitudes, Geophys. Res. Lett., 34, 13, https://doi.org/10.1029/2007GL029979, 2007.

Zhang, Q., Jimenez, J. L., Canagaratna, M. R., Ulbrich, I. M., Ng, N. L., Worsnop, D. R., and Sun, Y.: Understanding atmospheric organic aerosols via factor analysis of aerosol mass spectrometry: a review, Anal. Bioanal. Chem., 401, 3045-3067, https://doi.org/10.1007/s00216-011-5355-y, 2011. 